\title{
Research Square \\ Diurnal variations in the stratosphere of an ultrahot exoplanet
}

\section{Thomas Mikal-Evans ( $\nabla$ tmevans@mpia.de)}

Max Planck Institut fur Astronomie

\section{David Sing}

Johns Hopkins University https://orcid.org/0000-0001-6050-7645

Joanna Barstow

The Open University

\section{Tiffany Kataria}

Jet Propulsion Laboratory, California Institute of Technology

Jayesh Goyal

Cornell University

Nikole Lewis

Cornell University

Jake Taylor

University of Oxford

Nathan Mayne

University of Exeter https://orcid.org/0000-0001-6707-4563

Tansu Daylan

Massachusetts Institute of Technology

Hannah Wakeford

University of Bristol

Mark Marley

Univ. of Arizona https://orcid.org/0000-0002-5251-2943

Jessica Spake

California Institute of Technology

\section{Article}

Keywords: exoplanets, WASP-121b, spectroscopic phase curve

Posted Date: November 23rd, 2021

DOI: https://doi.org/10.21203/rs.3.rs-108192/v1 
License: (c) (i) This work is licensed under a Creative Commons Attribution 4.0 International License. Read Full License

Version of Record: A version of this preprint was published at Nature Astronomy on February 21st, 2022. See the published version at https://doi.org/10.1038/s41550-021-01592-w. 


\section{Diurnal variations in the stratosphere of an ultrahot exoplanet}

Thomas Mikal-Evans ${ }^{1,2} *$, David K. Sing ${ }^{3,4}$, Joanna K. Barstow ${ }^{5}$, Tiffany Kataria ${ }^{6}$, Jayesh Goyal ${ }^{7,8}$, Nikole Lewis ${ }^{7}$, Jake Taylor $^{9,10}$, Nathan. J. Mayne ${ }^{11}$, Tansu Daylan ${ }^{2,12}$, Hannah R. Wakeford ${ }^{13}$, Mark S. Marley ${ }^{14}$, Jessica J. Spake ${ }^{15}$

${ }^{1}$ Max-Planck-Institut für Astronomie, Heidelberg, Germany. ${ }^{2}$ Kavli Institute for Astrophysics and Space Research, Massachusetts Institute of Technology, Cambridge, MA, USA. ${ }^{3}$ Department of Earth \& Planetary Sciences, Johns Hopkins University, Baltimore, MD, USA. ${ }^{4}$ Department of Physics \& Astronomy, Johns Hopkins University, Baltimore, MD, USA. ${ }^{5}$ School of Physical Sciences, The Open University, Milton Keynes, UK. ${ }^{6}$ Jet Propulsion Laboratory, California Institute of Technology, Pasadena, CA, USA. ${ }^{7}$ Department of Astronomy and Carl Sagan Institute, Cornell University, Ithaca, NY, USA. ${ }^{8}$ National Institute of Science Education and Research (NISER), HBNI, Jatni, Odisha, India. ${ }^{9}$ Department of Physics, and Institute for Research on Exoplanets, Université de Montréal, Montreal, Canada. ${ }^{10}$ Department of Physics (Atmospheric, Oceanic and Planetary Physics), University of Oxford, Oxford, UK. ${ }^{11}$ Astrophysics Group, School of Physics and Astronomy, University of Exeter, Exeter, UK.

${ }^{12}$ Department of Astrophysical Sciences, Princeton University, Princeton, NJ, USA. ${ }^{13}$ School of Physics, University of Bristol, Bristol, UK. ${ }^{14}$ Lunar and Planetary Laboratory, Department of Planetary Sciences, Univ. of Arizona, Tucson, AZ, USA. ${ }^{15}$ Division of Geological and Planetary Sciences, California Institute of Technology, Pasadena, CA, USA.

*e-mail: tmevans@mpia.de 
1 The temperature profile of a planetary atmosphere is a key diagnostic of radiative and

2 dynamical processes governing the absorption, redistribution, and emission of energy.

3 Observations have revealed dayside stratospheres that either $\operatorname{cool}^{1,2}$ or warm $^{3,4}$ with altitude

4 for a small number of gas giant exoplanets, while others are consistent with constant

5 temperatures. ${ }^{5-8}$ Here we report spectroscopic phase curve measurements for the gas giant

6 WASP-121b, ${ }^{9}$ which constrain stratospheric temperatures throughout the diurnal cycle.

7 Variations measured for a water vapour spectral feature reveal a temperature profile that

8 transitions from warming with altitude on the dayside hemisphere to cooling with altitude

9 on the nightside hemisphere. The data are well explained by models assuming chemical

10 equilibrium, with water molecules thermally dissociating at low pressures on the dayside and

11 recombining on the nightside. ${ }^{10,11}$ Nightside temperatures are low enough for perovskite

$12\left(\mathrm{CaTiO}_{3}\right)$ to condense, which could deplete titanium from the gas phase ${ }^{12,13}$ and explain

13 recent non-detections at the day-night terminator. ${ }^{14-17}$ Nightside temperatures are also low

14 enough for refractory species, such as magnesium, iron, and vanadium, to condense.

15 Detections ${ }^{16-19}$ of these metals at the day-night terminator suggest, however, that if they do

16 form nightside clouds, cold trapping does not efficiently remove them from the upper

17 atmosphere.

18 WASP-121b is an ultrahot (>2,000 Kelvin) gas giant exoplanet orbiting an F6V star every 30.6

19 hours. ${ }^{9}$ Previous observations have shown that the dayside hemisphere of WASP-121b has a

20 thermal inversion, with a temperature profile that increases with increasing altitude or,

21 equivalently, with decreasing atmospheric pressure. ${ }^{3,20,21}$ The thermal inversion is thought to be

22 caused by the presence of optical absorbers capturing a significant fraction of incident stellar

23 radiation at low pressures in the atmosphere. ${ }^{22-24}$ Observations of the planet during transit 
24 geometry have identified a number of such absorbers, including gaseous $\mathrm{Fe}, \mathrm{Mg}, \mathrm{Cr}, \mathrm{V}$, and $\mathrm{VO} .{ }^{16-}$ $25 \quad 19$

Two full-orbit phase curves of WASP-121b were observed at epochs in 2018 and 2019

27 with the Hubble Space Telescope (HST) Wide Field Camera 3 (WFC3) infrared spectrograph. For

28 each observation, a time series of spectra was acquired using the G141 grism, which covers the

$291.12-1.64 \mu \mathrm{m}$ wavelength range. Further technical details of the observations are provided in

30 Methods. A broadband light curve was produced by summing each spectrum in the time series

31 across the full wavelength range (Extended Data Fig. 1). This light curve was fit by simultaneously

32 modelling the planet signal and instrumental systematics, as described in Methods. Quantitative

33 results are reported in Extended Data Table 1 and the best-fit model is shown in Fig. 1a, with

34 orbital phases of 0 and 0.5 corresponding to the primary transit and secondary eclipse mid-times,

35 respectively. As described in Methods, the best-fit phase curve model was inverted to generate a

36 global temperature map for WASP-121b (Fig. 1b). On the dayside hemisphere temperatures

37 exceed 3,000 Kelvin and drop to below 1,500 Kelvin in the coolest regions of the nightside

38 hemisphere.

39 To recover the planetary emission spectrum at different orbital phases, light curves were

40 generated for twelve spectroscopic channels across the 1.12-1.64 $\mu \mathrm{m}$ wavelength range (Extended

41 Data Fig. 2). These light curves were analysed using a similar method to the broadband light curve

42 fit (see Methods). The measured emission maxima of the spectroscopic phase curves give the

43 spectrum of the planetary dayside hemisphere, shown in Fig. 2a. In addition, phase-resolved

44 emission spectra were generated by averaging the planetary flux inferred from the spectroscopic

45 light curve fits across sixteen bins in orbital phase. The planetary emission spectrum recovered

46 immediately prior to the primary transit is shown in Fig. $2 \mathrm{~b}$ and is comprised almost entirely of 
47 emission from the nightside hemisphere of the planet. At intermediate phases, the emission

48 received from WASP-121b emanates from a combination of the dayside and nightside 49 hemispheres..$^{25-28}$

50 Wavelengths covered by the data are sensitive to an opacity band of $\mathrm{H}_{2} \mathrm{O}$ vapour and 51 continuum opacity of $\mathrm{H}^{-}$(Fig. 2). The measured shape and amplitude of these spectral features 52 allow the chemical abundances and vertical temperature profile of the atmosphere to be inferred. ${ }^{1-}$ $53{ }^{4}$ To recover these properties from the data, a retrieval analysis was first performed on the dayside 54 emission spectrum. As described in Methods, the overall heavy element enrichment ('metallicity') 55 of the atmosphere was allowed to vary, with the relative abundances of individual elements held 56 fixed to solar ratios, and a one-dimensional analytic temperature profile was adopted with three 57 free parameters. As the metallicity and temperature profile were varied in the fitting, chemical 58 abundances were computed assuming chemical equilibrium. Results of this analysis are reported 59 in Extended Data Table 2, including a measured metallicity of $[\mathrm{M} / \mathrm{H}]=0.76_{-0.62}^{+0.30}$ (approximately

60 1-10x solar). A second retrieval analysis was also performed for the nightside emission spectrum.

61 Due to the lower signal-to-noise, the atmospheric metallicity was held fixed to the value 62 determined from the dayside retrieval analysis, leaving only the three temperature profile 63 parameters free. As described in Methods, the contribution to the overall emission from the narrow 64 crescent of dayside hemisphere visible at this phase was also factored in to the modelled emission. 65 The inferred dayside and nightside emission spectra are shown in Fig. 2 and the 66 corresponding pressure-dependent temperatures, $\mathrm{H}_{2} \mathrm{O}$ abundances, $\mathrm{H}^{-}$abundances, and 67 contribution functions are shown in Fig. 3. A dayside thermal inversion is inferred at the pressures 68 probed by the data (below $\sim 30$ millibar), consistent with previous results. ${ }^{3,20,21}$ On the dayside, the $69 \mathrm{H}_{2} \mathrm{O}$ abundance drops sharply with decreasing pressure, due to thermal dissociation of 
70 molecules. ${ }^{10,11}$ Thermal ionisation also raises the abundance of free electrons, which bind with

71 atomic hydrogen to form $\mathrm{H}^{-}$(refs 7-11, 29). As temperatures decrease on the nightside, $\mathrm{H}_{2} \mathrm{O}$

72 molecules recombine at low pressures. Rotational-vibrational transitions of $\mathrm{H}_{2} \mathrm{O}$ molecules at near-

73 infrared wavelengths increase the efficiency of radiative cooling in the upper atmosphere

74 (Extended Data Fig. 3), resulting in temperature profiles that cool with decreasing pressure on the

75 nightside (Fig. 3a). As described in Methods, consistent results for the dayside and nightside

76 hemisphere properties were obtained when retrievals were performed at intermediate phases

77 (Extended Data Figs 4-7) and when the assumption of chemical equilibrium was relaxed (Figs 2

78 and 3, Extended Data Fig. 8).

79 These measurements provide empirical constraints for the theory that refractory species

80 may be lost from the upper atmosphere of highly-irradiated planets due to cold trap processes. ${ }^{12}$

81 For example, due to the high temperature contrasts expected between the dayside and nightside

82 hemispheres, refractory species could condense on the nightside and settle to deeper layers of the

83 atmosphere, despite dayside temperatures being high enough to maintain them in the gas phase.

84 However, day-night cold trapping of this kind might be avoided if vertical mixing is vigorous

85 within the atmosphere, allowing condensates to be suspended aloft long enough for lateral winds

86 to return them to the dayside hemisphere. ${ }^{30,31}$ Alternatively, condensates may gravitationally settle

87 to deeper layers of the atmosphere and subsequently re-enter the gas phase as they are returned to

88 lower pressures by updrafts. ${ }^{13}$

89 Condensation curves for relevant refractory species ${ }^{31-33}$ are shown in Fig. 3a, namely,

90 corundum $\left(\mathrm{Al}_{2} \mathrm{O}_{3}\right)$, perovskite $\left(\mathrm{CaTiO}_{3}\right), \mathrm{VO}, \mathrm{Fe}$, forsterite $\left(\mathrm{Mg}_{2} \mathrm{SiO}_{4}\right)$, and enstatite $\left(\mathrm{MgSiO}_{3}\right)$.

91 The corundum, perovskite, and Fe condensation curves are crossed during the WASP-121b diurnal

92 cycle (Fig. 3a) and it is also likely that those of forsterite, VO, and enstatite are crossed in the 
93 coolest regions of the nightside hemisphere (Fig. 1b). It is particularly significant that temperatures

94 drop low enough for $\mathrm{Fe}, \mathrm{Ca}, \mathrm{Mg}$, and $\mathrm{V}$ to condense, as recent observations have revealed these

95 heavy metals in the gas phase at the day-night terminator. ${ }^{16-19}$ Vertical mixing must therefore be

96 operating efficiently within the atmosphere of WASP-121b, to avoid day-night cold trapping. This

97 also appears to be the case for another ultrahot gas giant, WASP-76b, for which gaseous Fe has

98 been detected at the eastern terminator but not detected at the cooler western terminator, where it

99 has presumably condensed. ${ }^{34}$ However, non-detections of $\mathrm{Ti}$ and $\mathrm{TiO}$ at the day-night terminator

100 of WASP-121b complicate this picture, ${ }^{14-17}$ as these gases should also form condensates such as

101 perovskite and $\mathrm{TiO}_{2}$ on the nightside. ${ }^{31-33}$ It would be surprising if Ti-bearing condensates are

102 efficiently cold trapped while other refractory species avoid a similar fate. This is especially true

103 for $\mathrm{V}$, which is chemically similar to Ti but an order of magnitude less abundant in the solar

104 neighbourhood. ${ }^{35,36}$ For now, this remains an outstanding puzzle, with a solution that may depend

105 on additional factors such as variations in surface energies between different condensate species. ${ }^{37}$

106 The dayside and nightside emission spectra predicted by a cloud-free three-dimensional

107 general circulation model (GCM) simulation generated for this study (Methods) and results from

108 two published $\mathrm{GCMs}^{10}$ are shown in Fig. 2. Good agreement with the data is obtained, suggesting

109 that the GCMs have successfully captured much of the interplay between the radiation, chemistry,

110 and dynamics of the WASP-121b atmosphere. The broadband phase curve predicted by the GCM

111 simulation run for this study is also shown in Fig. 1, having an overall amplitude in respectable

112 agreement with the data. However, around the quadrature phases (i.e. 0.25 and 0.75 ), the GCM

113 underpredicts the planetary emission (see also Extended Data Figs 2 and 4). Nightside clouds are

114 unlikely to explain this discrepancy, as they would be expected to lower the emission by blocking

115 radiation from deeper, warmer layers of the atmosphere. Refractory clouds forming close to the 
116 terminator region, however, could potentially boost the emission received from the dayside

117 crescent by reflecting light from the host star. ${ }^{21,38}$ Another possible explanation may be provided

118 by the optically thick exosphere of WASP-121b that has been observed to extend to the planet's

119 Roche limit, ${ }^{18}$ well below the pressure range considered by the GCMs. Heated layers of the stellar-

120 facing exosphere would be maximally visible at quadrature, raising the overall emission received

121 from the planet, whereas at superior and inferior conjunction, the data are sensitive to deeper

122 atmospheric layers due to the zenith viewing geometry (Fig. 3d), and as such are well matched by

123 the GCM predictions (Fig. 2). Furthermore, the GCMs did not include opacities for gaseous metals

124 such as Fe and Mg, which are known to be present in the atmosphere of WASP-121b $\mathrm{b}^{16-19}$ and could

125 contribute significantly to the outgoing emission. ${ }^{11}$ These effects, along with others not considered

126 here, such as latent heat release from the dissociation/recombination of hydrogen ${ }^{29}$ and

127 atmospheric drag, ${ }^{8,39}$ should be investigated in future modelling.

128 The dynamics and chemistry of ultrahot gas giants such as WASP-121b are exotic by solar

129 system standards, driven by dramatic contrasts in the irradiation environments of the dayside and

130 nightside hemispheres. Until now, it has proven challenging to explore these diurnal variations due

131 to the narrow infrared wavelength coverage of HST. ${ }^{8,40}$ For WASP- $121 \mathrm{~b}$, these wavelengths are

132 fortuitously sensitive to a pressure range that allows the transition from inverted to non-inverted

133 temperature profiles to be mapped globally. Further insights are anticipated with the James Webb

134 Space Telescope, which will enable higher signal-to-noise spectroscopy across the broader 0.8-

$13511 \mu \mathrm{m}$ wavelength range. This will provide fuller coverage of the $\mathrm{H}^{-}$opacity continuum and access

136 to stronger $\mathrm{H}_{2} \mathrm{O}$ bands at longer wavelengths, breaking the degeneracy between the two species.

137 Additional spectral features, such as the $\mathrm{CO}$ spectral band at $4.5 \mu \mathrm{m}$, will provide further leverage

138 for constraining the chemical composition, thermal structure, and wind patterns of the atmosphere. 


\section{References}

1. Stevenson, K. B. et al. Thermal structure of an exoplanet atmosphere from phase-resolved emission spectroscopy. Science. 346, 838 (2014).

2. Line, M. R. et al. No Thermal Inversion and a Solar Water Abundance for the Hot Jupiter HD 209458b from HST/WFC3 Spectroscopy. Astron. J. 152, 203 (2016).

3. Mikal-Evans, T. et al. Confirmation of water emission in the dayside spectrum of the ultrahot Jupiter WASP121b. Mon. Not. R. Astron. Soc. 496, 1638 (2020).

4. Nugroho, S. K. et al. High-resolution Spectroscopic Detection of TiO and a Stratosphere in the Day-side of WASP-33b. Astron. J. 154, 221 (2017).

5. Nikolov, N. et al. Hubble PanCET: an isothermal day-side atmosphere for the bloated gas-giant HAT-P-32Ab. Mon. Not. R. Astron. Soc. 474, 1705 (2018).

6. Mansfield, M. et al. An HST/WFC3 Thermal Emission Spectrum of the Hot Jupiter HAT-P-7b. Astron. J. 156, 10 (2018).

7. Arcangeli, J. et al. H- Opacity and Water Dissociation in the Dayside Atmosphere of the Very Hot Gas Giant WASP-18b. Astrophys. J. 855, L30 (2018).

8. Kreidberg, L. et al. Global Climate and Atmospheric Composition of the Ultra-hot Jupiter WASP-103b from HST and Spitzer Phase Curve Observations. Astron. J. 156, 17 (2018).

9. Delrez, L. et al. WASP-121 b: a hot Jupiter close to tidal disruption transiting an active F star. Mon. Not. $R$. Astron. Soc. 458, 4025 (2016).

10. Parmentier, V., Fortney, J. J., Showman, A. P., Morley, C., Marley, M. S. From thermal dissociation to condensation in the atmospheres of ultra hot Jupiters: WASP-121b in context. Astron. Astrophys. 617, A110 (2018).

11. Lothringer, J. D., Barman, T., Koskinen, T. Extremely Irradiated Hot Jupiters: Non-oxide Inversions, H- Opacity, and Thermal Dissociation of Molecules. Astrophys. J. 866, 27 (2018).

12. Spiegel, D. S., Silverio, K., Burrows, A. Can TiO Explain Thermal Inversions in the Upper Atmospheres of Irradiated Giant Planets? Astrophys. J. 699, 1487 (2009).

13. Parmentier, V., Showman, A. P., Lian, Y. 3D mixing in hot Jupiters atmospheres. I. Application to the day/night cold trap in HD 209458b. Astron. Astrophys. 558, A91 (2013). 
14. Evans, T. M. et al. An Optical Transmission Spectrum for the Ultra-hot Jupiter WASP-121b Measured with the Hubble Space Telescope. Astron. J. 156, 283 (2018).

15. Merritt, S. R. et al. Non-detection of $\mathrm{TiO}$ and $\mathrm{VO}$ in the atmosphere of WASP-121b using high-resolution spectroscopy. Astron. Astrophys. 636, A117 (2020).

16. Ben-Yami, M. et al. Neutral Cr and V in the Atmosphere of Ultra-hot Jupiter WASP-121 b. Astrophys. J. 897, L5 (2020).

17. Hoeijmakers, H. J. et al. Hot Exoplanet Atmospheres Resolved with Transit Spectroscopy (HEARTS). IV. A spectral inventory of atoms and molecules in the high-resolution transmission spectrum of WASP-121 b. Astron. Astrophys. 641, A123 (2020).

18. Sing, D. K. et al. The Hubble Space Telescope PanCET Program: Exospheric Mg II and Fe II in the Nearultraviolet Transmission Spectrum of WASP-121b Using Jitter Decorrelation. Astron. J. 158, 91 (2019).

19. Gibson, N. P. et al. Revisiting the potassium feature of WASP-31b at high resolution. Mon. Not. R. Astron. Soc. 482, 606 (2019).

20. Bourrier, V. et al. Optical phase curve of the ultra-hot Jupiter WASP-121b. Astron. Astrophys. 637, A36 (2020).

21. Daylan, T. et al. TESS observations of the WASP-121 b phase curve. Astron. J. 161, 131 (2020).

22. Hubeny, I., Burrows, A., Sudarsky, D. A Possible Bifurcation in Atmospheres of Strongly Irradiated Stars and Planets. Astrophys. J. 594, 1011 (2003).

23. Burrows, A., Hubeny, I., Budaj, J., Knutson, H. A., Charbonneau, D. Theoretical Spectral Models of the Planet HD 209458b with a Thermal Inversion and Water Emission Bands. Astrophys. J. 668, L171 (2007).

24. Fortney, J. J., Lodders, K., Marley, M. S., Freedman, R. S. A Unified Theory for the Atmospheres of the Hot and Very Hot Jupiters: Two Classes of Irradiated Atmospheres. Astrophys. J. 678, 1419 (2008).

25. Feng, Y. K., Line, M. R., Fortney, J. J. 2D retrieval frameworks for hot Jupiter phase curves. Astron. J. 169, 137 (2020).

26. Taylor, J. et al. Understanding and mitigating biases when studying inhomogeneous emission spectra with JWST. Mon. Not. R. Astron. Soc. 493, 4342 (2020).

27. Irwin, P. G. J. et al. 2.5 retrieval of atmospheric properties from exoplanet phase curves: application to WASP43b observations. Mon. Not. R. Astron. Soc. 493, 106 (2020). 
28. Changeat, Q., Al-Refaie, A. TauREx3 PhaseCurve: a 1.5D model for phase-curve description. Astrophys. J. 898, 155 (2020).

29. Bell, T. J., Cowan, N. B. Increased Heat Transport in Ultra-hot Jupiter Atmospheres through H- Dissociation and Recombination. Astrophys. J. 857, L20 (2018).

30. Heng, K., Demory, B-.O. Understanding Trends Associated with Clouds in Irradiated Exoplanets. Astrophys. J. 777,100 (2013).

31. Helling, C. et al. Understanding the atmospheric properties and chemical composition of the ultra-hot Jupiter HAT-P-7b. I. Cloud and chemistry mapping. Astron. Astrophys. 631, A79 (2019).

32. Burrows, A., Sharp, C. M. Chemical Equilibrium Abundances in Brown Dwarf and Extrasolar Giant Planet Atmospheres. Astrophys. J. 512, 843 (1999).

33. Wakeford, H. R. et al. High-temperature condensate clouds in super-hot Jupiter atmospheres. Mon. Not. R. Astron. Soc. 464, 4247 (2017).

34. Ehrenreich, D. et al. Nightside condensation of iron in an ultrahot giant exoplanet. Nature. 580,597 (2020).

35. Lodders, K. Titanium and Vanadium Chemistry in Low-Mass Dwarf Stars. Astrophys. J. 577, 974 (2002).

36. Asplund, M., Grevesse, N., Sauval, A. J., Scott, P. The Chemical Composition of the Sun. Annual Review of Astron. Astrophys. 47, 481 (2009).

37. Gao, P. et al. Aerosol composition of hot giant exoplanets dominated by silicates and hydrocarbon hazes. Nature Astronomy. 4, 951 (2020).

38. Parmentier, V. et al. Transitions in the cloud compositions of hot Jupiters. Astrophys. J. 828, 22 (2016).

39. Komacek, T. D., Showman, A. P., Tan, X. Atmospheric circulation of hot Jupiters: dayside-nightside temperature differences. II. Comparison with observations. Astrophys. J. 835, 198 (2017).

40. Arcangeli, J. et al. Climate of an ultra hot Jupiter. Spectroscopic phase curve of WASP-18b with HST/WFC3. Astron. Astrophys. 625, A136 (2019). 


\section{Figures}

a
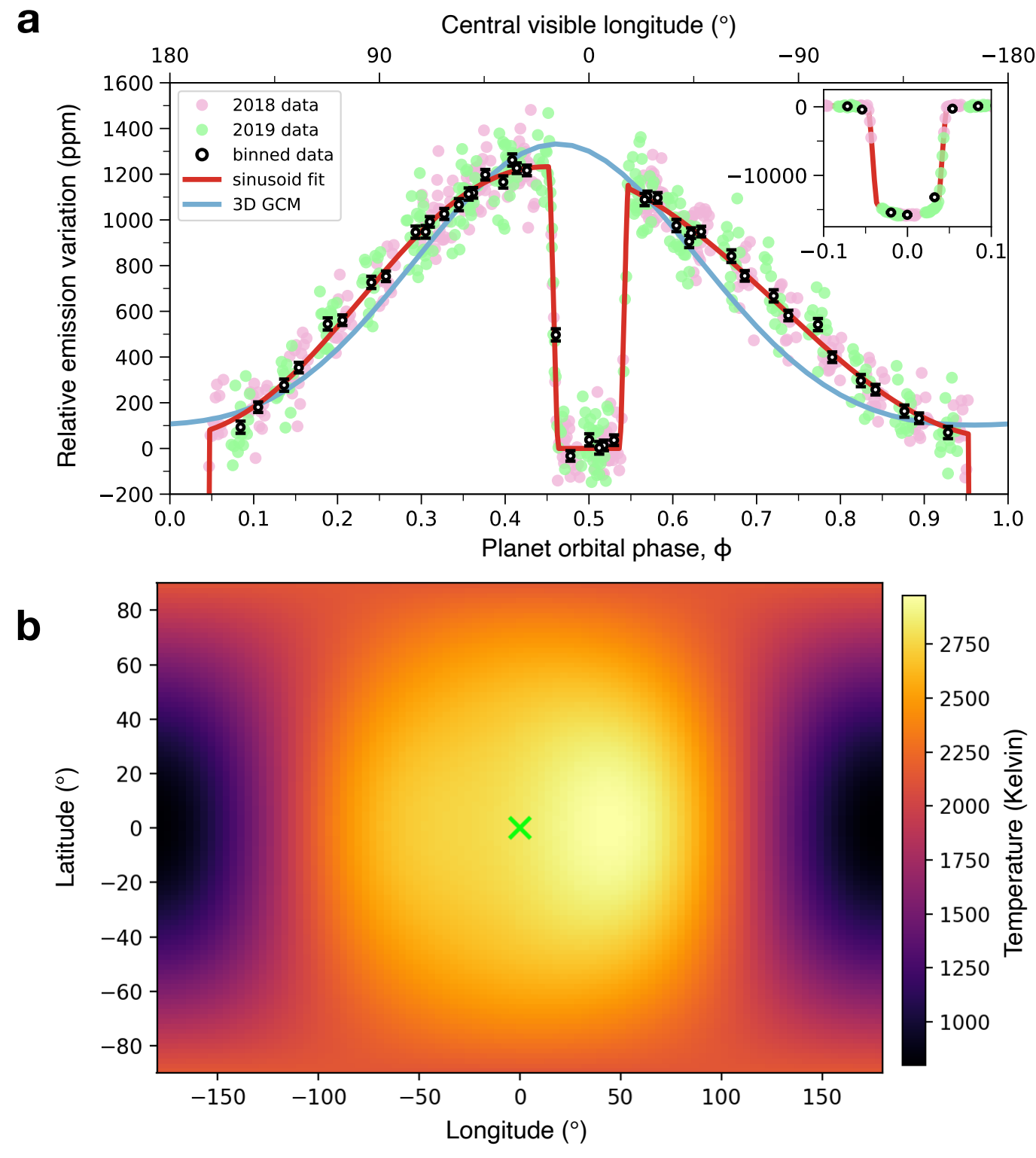

Fig. 1. Broadband phase curve and inverted temperature map for WASP-121b. a, Planet emission relative to the host star emission as a function of the planetary orbital phase. Pink and green circles show WFC3 measurements made at epochs in 2018 and 2019, respectively. Black circles show binned data for individual HST orbits, with error bars indicating the $1 \sigma$ measurement uncertainties. Red line shows the maximum likelihood second-order sinusoidal model, including primary transit and secondary eclipse signals. Blue line shows the prediction of a 3D GCM simulation. Inset shows the full primary transit signal. b, Latitude-longitude temperature map obtained by inverting the maximum likelihood phase curve model as described in Methods. Green cross indicates the location of the substellar point. 


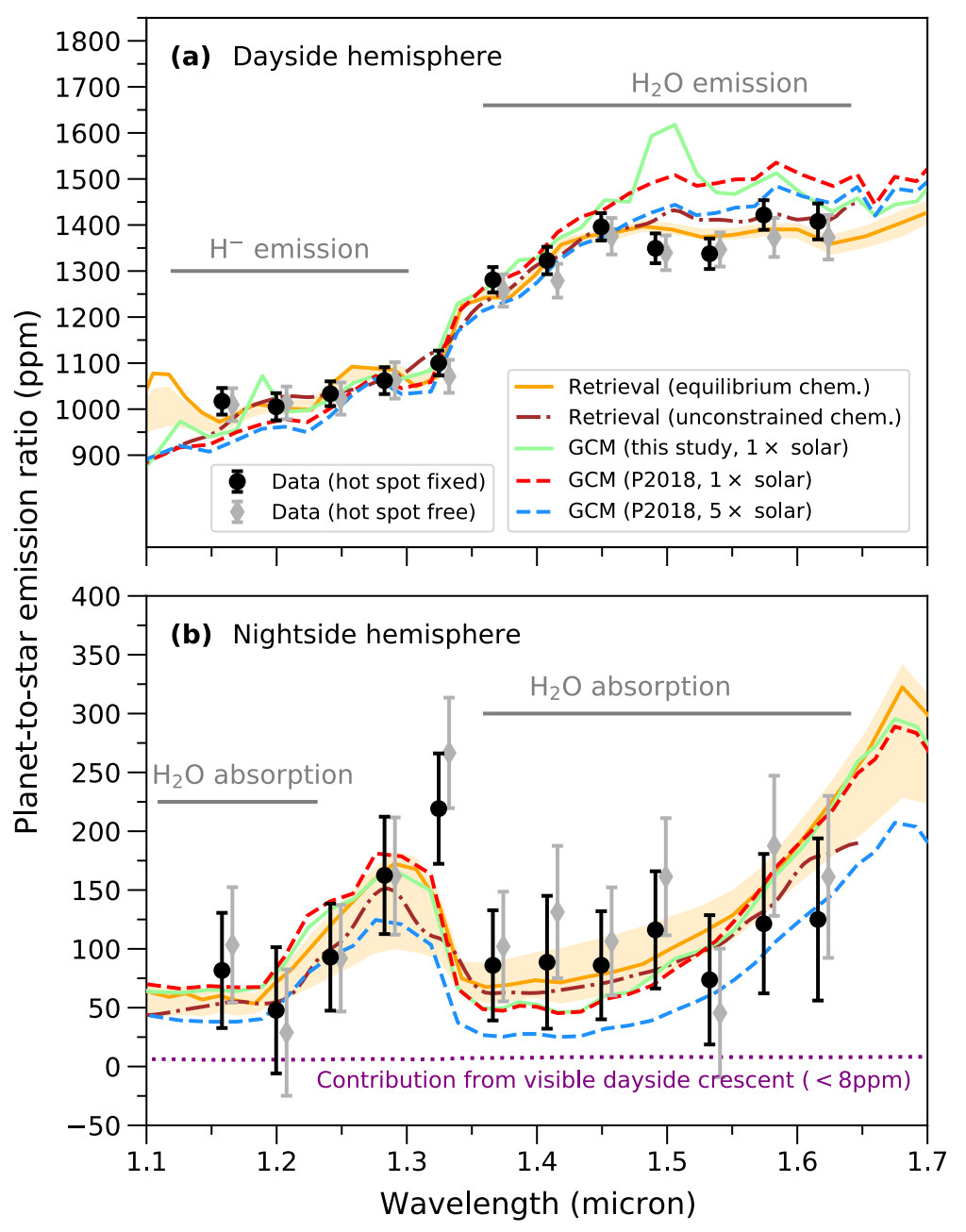

Fig. 2. Emission spectra for the dayside and nightside hemispheres of WASP-121b. a, Black circles show measured dayside emission with error bars corresponding to $1 \sigma$ measurement uncertainties. Grey diamonds show the same, but for a light curve analysis in which the phase of maximum brightness ('hot spot') was allowed to vary in each spectroscopic channel (see Methods) and with small horizontal offsets applied for visual clarity. Orange solid line shows the maximum likelihood model and yellow shading shows the $1 \sigma$ credible range of model predictions from the ATMO retrieval analysis assuming chemical equilibrium. Brown dot-dashed line shows the maximum likelihood model from the NEMESIS retrieval analysis with unconstrained chemistry (see Methods). Light green solid line shows the prediction of the 3D GCM run for this study. Dashed red and blue lines show predictions of the 3D GCMs for WASP-121b published in ref. 13 assuming metallicities of $1 \mathrm{x}$ and $5 \mathrm{x}$ solar, respectively. $\mathbf{b}$, The same as a, but showing results for the nightside hemisphere emission obtained at orbital phase 0.95 , immediately prior to primary transit ingress. Dotted purple line also shows the emission contribution from the narrow crescent of dayside hemisphere visible at this orbital phase, which does not exceed 8ppm across the wavelengths covered by the data. 

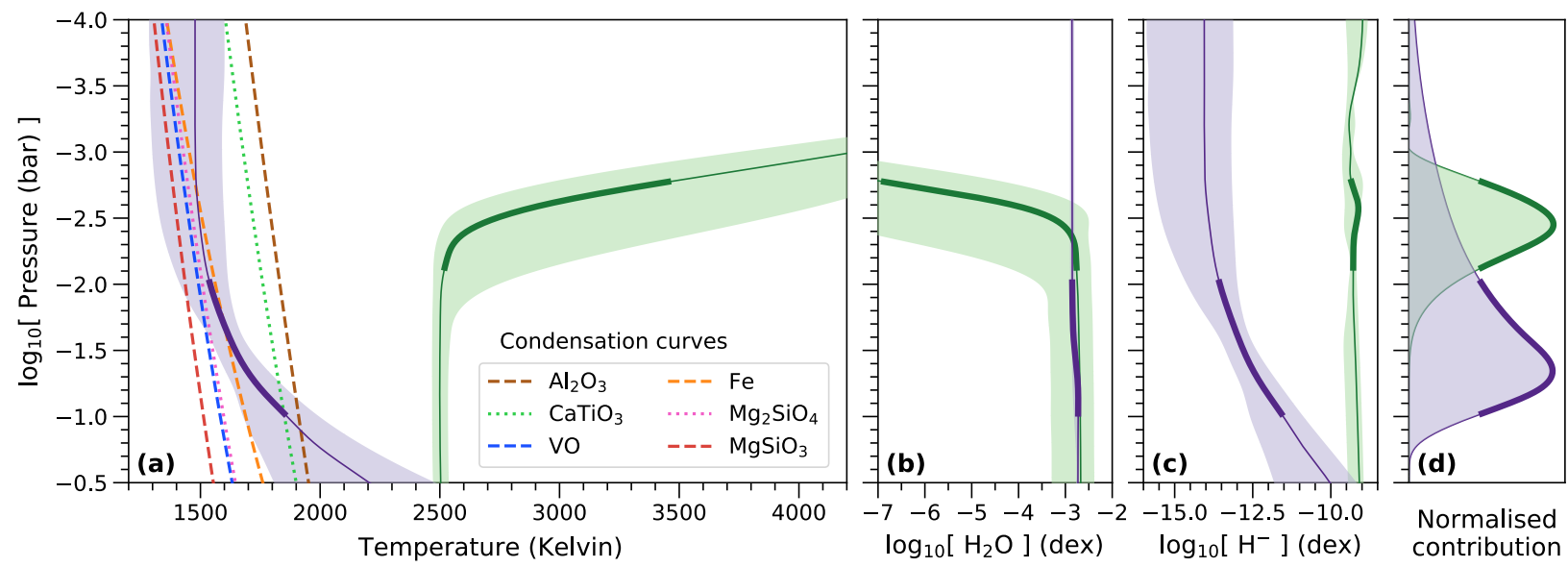

Fig. 3. Pressure-dependent atmospheric properties retrieved for WASP-121b. a, Green solid line shows the median temperature at each pressure level inferred for the dayside hemisphere by the ATMO retrieval analysis, with green shading showing the $1 \sigma$ credible range. Purple solid line and purple shading show the same, but for the nightside hemisphere. Thick sections of the purple and green lines indicate the pressure levels from which the majority of the planetary emission is emanating at the wavelengths covered by the data. b, c, Vertical abundances for $\mathrm{H}_{2} \mathrm{O}$ and $\mathrm{H}^{-}$, using the same colour scheme as panel a. The narrow range of allowed $\mathrm{H}_{2} \mathrm{O}$ abundances for the nightside is a result of the fixed metallicity. For $\mathrm{H}^{-}$, the nightside abundance is more uncertain than for the dayside, because no $\mathrm{H}^{-}$spectral features are detected in the nightside spectrum. However, the $\mathrm{H}^{-}$abundance can still vary under the assumption of chemical equilibrium within the range of allowed temperatures, as the latter mediates the abundance of free electrons through thermal ionisation. d, Normalised contribution functions for the dayside and nightside hemispheres, integrated across the wavelength range covered by the data, using the same colour scheme as panel $\mathbf{a}$. 


\section{METHODS}

\section{Observations and data reduction}

140 Two full orbit phase curves of WASP-121b were observed on 2018 March 12-13 and 2019

141 February 3-4 using the Hubble Space Telescope (HST) Wide Field Camera 3 (WFC3) infrared

142 spectrograph with the G141 grism, covering a wavelength range of approximately $1.12-1.64 \mu \mathrm{m}$

143 micron. Each observation was performed over 26 consecutive HST orbits and lasted approximately

$14440.3 \mathrm{hr}$. The timing of each observation was designed to encompass two consecutive secondary

145 eclipses, as these correspond to times when only the star is visible, allowing the baseline flux level

146 to be calibrated at the beginning and end of the observation. Furthermore, both observations were

147 scheduled such that the combined dataset provided maximum phase coverage for the planetary

148 orbit, which has a period of $30.6 \mathrm{hr}$. Due to the long duration of the observations, full guide star

149 reacquisition sequences were performed at the beginning of the 10th and 20th HST orbits.

150 Integration times per exposure were $103 \mathrm{sec}$ over 15 non-destructive reads (NSAMP=15) using

151 the SPARS10 sampling sequence. Science exposures were made using the spatial scanning mode

152 with scans along approximately 60 pixel rows of the detector cross-dispersion axis at a rate of

$1530.073 \operatorname{arcsec~} \sec ^{-1}$. With this setup, 415 exposures were acquired for each phase curve observation.

154 Peak frame counts were kept below $\sim 40,000$ electrons pixel $^{-1}$, within the recommended range for 155 the detector. ${ }^{41}$

156 Spectra were extracted from each data frame using a custom Python code. ${ }^{3,42-44}$ In brief,

157 this involved first estimating the background flux for each exposure by taking the median count 158 within a $10 \times 170$ pixel box away from the target on the detector. The background was then 159 subtracted from each exposure and the target flux summed along the cross-dispersion axis within 
160 a rectangular aperture spanning 100 pixel rows, giving the target flux as a function of location

161 along the detector dispersion axis. The mapping from the dispersion axis to wavelength was

162 determined by cross-correlating these measured fluxes against a model stellar spectrum modulated

163 by the G141 grism throughput.

165 Broadband light curve analysis

166 Broadband light curves were produced by integrating the time series of target spectra across the 167 dispersion axis and are shown in Extended Data Fig. 1. The primary transits, secondary eclipses, 168 and phase curve variations for the planet are easily visible by eye. However, the data are also 169 affected by instrumental systematics caused by charge-trapping on the detector, producing a ramp170 like trend in each HST orbit. In addition, longer-term instrumental drifts are evident, particularly 171 at the beginning of the observations, and subtle discontinuities affect the measured flux at the HST 172 orbits immediately following guide star reacquisitions.

173 We modelled both light curves jointly to extract properties of the planet. Our overall model $174 \quad M$ took the form:

$175 \quad M_{k j}=B_{k j} \cdot \Psi_{k} \cdot \Pi$

176 where $B$ is the instrumental baseline trend, $\Psi$ is the detector ramp systematic, and $\Pi$ is the 177 astrophysical signal. Here, the ' $k$ ' subscripts refer to the observation number ( $k=1$ for the 2018 178 observation and $k=2$ for the 2019 observation) and the ' $j$ ' subscripts refer to the data segment 179 number. For the latter, we divided each observation into three data segments, defined by the guide 180 star re-acquisitions (Extended Data Fig. 1). In summary, we modelled: three data segments with 181 independent baseline trends, for each observation; ramp systematics separately for each 
182 observation but shared across data segments; and a planet signal shared across all data segments 183 of both observations.

184 For $B_{k j}$, we followed standard practice 8,40 and adopted a quadratic trend in time $t$ for the 185 first data segment $(j=1)$ of each visit. For the second $(j=2)$ and third $(j=3)$ data segments, we 186 adopted linear $t$ trends. This was done because the baseline drift is clearly less pronounced 187 following the first data segment (Extended Data Fig. 1), as the spacecraft and instrument has settled 188 into a stable configuration. We also tested quadratic $t$ trends for $j=2$ and $j=3$, but found this $\operatorname{did}$ not 189 improve the quality of the fit, justifying the use of the simpler linear $t$ trends.

190 For $\Psi_{k}$, we adopted the analytic treatment of ref. 45 , which is motivated by a simple model 191 of electron charge-trapping on the detector. Explicitly:

$\Psi 92=r(t, \tau)=r$

193 where $\tau$ is the time from the start of each HST orbit, and:

$$
\begin{aligned}
& r=1+a_{1} \cdot \exp \left[-t / a_{2}\right] \\
& \rho=1+a_{3} \cdot \exp \left[-(t-\tau) /\left(a_{4} \cdot r\right)\right]
\end{aligned}
$$

197 system:

$$
\Pi=F_{s}+F_{p},
$$

199 where $F_{s}$ is the emission from the host star and $F_{p}$ is the emission from the planet. The stellar flux $200 F_{s}$ is assumed to be constant except when the planet transits in front of the host star. We used a 201 publicly available software package ${ }^{46}$ to compute the drop in $F_{s}(t)$ during primary transit. We 202 modelled the planet emission signal as:

$$
F_{p}=[\Phi+\Gamma] \cdot E,
$$


204 where $\Phi$ denotes the phase variations, $\Gamma$ is the drop in flux received from the planet during 205 secondary eclipse, and $E$ are ellipsoidal variations caused by tidal distortion of the planet. For $\Phi$, 206 we used a second-order cosine function:

$$
\Phi=c_{0}+\left(c_{1} / 2\right) \cdot\left[1-\cos \left(\phi-c_{2}\right)\right]+\left(c_{3} / 2\right) \cdot\left[1-\cos \left(2 \phi-c_{4}\right)\right],
$$

208 where $\phi=2 \pi \cdot\left(t-T_{p}\right) / P$ is the planetary orbital phase and $T_{p}$ is the time of mid-transit. To compute $209 \Gamma$, we used the same publicly available software used for the primary transit signal. ${ }^{46}$ For $E$, we 210 used a cosine function of the form:

$$
E=1+\left(\varepsilon_{0} / 2\right) \cdot[1-\cos (2 \phi)],
$$

212 giving maximum cross-sectional area at orbital quadrature.

213 Using the above model, we defined a log-likelihood function for the joint dataset of the 214 form:

$$
\log P=\log N\left(\boldsymbol{y}_{1}-\boldsymbol{M}_{1}, K_{1}\right)+\log N\left(\boldsymbol{y}_{2}-\boldsymbol{M}_{2}, K_{2}\right)
$$

216 where $N(\boldsymbol{\mu}, K)$ denotes a multivariate normal distribution with mean vector $\boldsymbol{\mu}$ and covariance

217 matrix $K$. In Eq. 9, the mean vector is given by the $t$-dependent model residuals, where $\boldsymbol{y}_{k}$ is a 218 vector containing the data points and $\boldsymbol{M}_{k}$ is a vector containing the corresponding model values for 219 the $k$ th observation. The covariance matrices for each observation are assumed to be diagonal, with 220 the form $K_{k k}=\beta_{k} \sigma_{k} I$, where $\sigma_{k}$ is the photon noise value, $\beta_{k}$ is a white noise rescaling factor, and $I$ is 221 the identity matrix.

222 For the systematics components, our free parameters were the coefficients for the $t$ 223 dependent baseline trends $\left(B_{k}\right)$; the $a_{1, k}, a_{2, k}, a_{3, k}$, and $a_{4, k}$ parameters for the detector ramps $\left(\Psi_{k}\right)$; 224 and the white noise rescaling factors $\beta_{k}$. For the primary transit (i.e. $F_{s}$ ), the following parameters 225 were allowed to vary: planet-to-star radius ratio $\left(R_{p} / R_{\star}\right)$; normalised semimajor axis $\left(a / R_{\star}\right)$; orbital 226 impact parameter $\left(b=a \cdot \cos i / R_{\star}\right.$, where $i$ is the orbital inclination); and the primary transit mid- 
227 times $\left(T_{p}\right)$. These parameters were shared across both datasets, except for $T_{p}$ which was allowed to 228 vary separately for each dataset. Since the planetary orbital period has been previously determined 229 to a high level of precision, it was fixed to $P=1.2749247646$ day. ${ }^{18}$ A circular orbit was assumed, 230 given constraints from previous measurements. ${ }^{9}$ A quadratic stellar limb darkening profile was 231 adopted with coefficients $\left(u_{1}, u_{2}\right)$ fixed to values determined using a model of the host star 232 atmosphere, as described previously. For the phase variations $\Phi$, the parameters $c_{0}, c_{1}, c_{2}, c_{3}$, and $233 c_{4}$ were allowed to vary. For $\Gamma$, the secondary eclipse mid-time $T_{s}$ was linked to the primary transit 234 mid-time $T_{p}$ according to $T_{s}=T_{p}+P / 2$, given the assumed circular orbit. Rather than treating the 235 eclipse depth as a separate free parameter, it was constrained such that $F_{p}=0$ at the bottom of 236 eclipse. The ellipsoidal variation in the planetary cross-sectional area, $\varepsilon_{0}$, was also treated as a free 237 parameter. Uniform priors were adopted for all free parameters. Marginalisation of the posterior 238 distribution was performed using affine-invariant Markov chain Monte Carlo (MCMC) with 300 239 walkers and 1,600 steps, as implemented by a publicly available software package. ${ }^{47}$ The best-fit 240 model is shown in Fig. 1a and the results for the astrophysical model parameters are summarised 241 in Extended Data Table 1.

242 In addition, a simpler first-order sinusoidal model was tested, equivalent to fixing $c_{3}=c_{4}$

$243=0$ in Eq. 7. The results of this fit are also reported in Extended Data Table 1, with good agreement

244 obtained for parameters common to both models. However, the first-order sinusoidal model has a 245 significantly higher Bayesian Information Criterion (BIC) value of 2129.5 compared to 2111.0 for 246 the second-order sinusoidal model, corresponding to a Bayes factor of $\exp (-\Delta \mathrm{BIC} / 2)=10^{4}$ in 247 favour of the second-order sinusoidal model. This provides strong evidence for asymmetry in the 248 phase curve about the 'hot spot' (i.e. phase of peak emission), which is better accounted for by the 
second-order sinusoidal model than by the first-order sinusoidal model. For this reason, the

250 second-order sinusoidal model is adopted as the preferred fit for the broadband light curve.

252 and second-order sinusoidal model fits. These were derived by computing $A_{F}=\left(F_{\max }-F_{\min }\right) / F_{\max }$

253 for each phase curve model sampled, where $F_{\max }$ and $F_{\min }$ are the maximum and minimum values

254 of the phase curve. The obtained value for $A_{F}(95.1 \pm 2.5 \%$ for the preferred second-order sinusoid 255 and $98.6 \pm 1.9 \%$ for the first-order sinusoid) is broadly in line with those reported for the three other 256 gas giant exoplanets with published WFC3 phase curve measurements: $A_{F}=100.5 \pm 1.3 \%$ for 257 WASP- $43 b,{ }^{1,48} A_{F}=91 \pm 2 \%$ for WASP- $103 b,{ }^{8}$ and $A_{F}>96 \%$ for WASP- $18 b .{ }^{40}$

258 A spherical harmonic of degree $l=2$ was also used to generate a temperature map of the 259 planet. The temperature map was converted to a corresponding phase curve signal in the G141 260 passband using a publicly available code. ${ }^{49}$ Coefficients were adjusted to optimise the match of 261 the resulting phase curve with the second-order sinusoidal function derived from the light curve 262 fits (Extended Data Fig. 9). Since the available phase curve data do not constrain latitudinal 263 temperature variations, coefficients of order $m=0$ were fixed to zero. The resulting temperature 264 map is shown in Fig. 1b. At longitudes approximately $9^{\circ}$ eastward of the substellar point, the 265 atmosphere reaches its highest temperatures of around 3,200 Kelvin. On the nightside hemisphere, 266 the coldest regions of the atmosphere are around 1,200 Kelvin, cool enough for numerous 267 refractory species to condense (Fig. 3a).

\section{Spectroscopic light curve analysis}

270 Spectroscopic light curves were generated by binning the spectra into twelve wavelength channels.

271 Before doing this, systematics common to all wavelengths were corrected using a cross-correlation 
272 technique $e^{3,42-44}$ based on an original implementation by ref. 48. This common-mode correction 273 effectively cleaned the detector ramp systematics in all but the first HST orbit of both datasets. It 274 also significantly reduced the baseline trend systematics due to instrumental drift.

275 Since the common-mode correction successfully removed most of the systematics affecting 276 each spectroscopic channel, a simpler model than described above for the broadband analysis was 277 adopted for the light curve fitting. Rather than fit a quadratic time trend for the instrumental 278 baseline of the first data segments (i.e. the $B_{k 1}$ terms of Eq. 1), linear time trends were used for all 279 data segments. Furthermore, with the exception of the first HST orbit of each dataset, a model for 280 the detector ramp was unnecessary. We therefore opted to discard the first HST orbit from each 281 spectroscopic light curve and effectively set $\Psi_{k}=1$ (Eq. 1) for the remaining orbits. The 282 astrophysical signal was modelled using the same model $\Pi$ as was used for the broadband light 283 curve fit. However, for the spectroscopic light curve fits, a number of parameters were held fixed 284 to the best-fit values determined from the broadband light curve (Extended Data Table 1), namely: $285 T_{p}, a / R_{s}$, and $b$, which do not vary with wavelength, and the ellipsoidal variation amplitude, $\varepsilon_{0}$. 286 Quadratic limb darkening coefficients $\left(u_{1}, u_{2}\right)$ were determined for each spectroscopic channel as 287 for the broadband light curve, and also held fixed during fitting. Aside from these details, light 288 curve fitting proceeded as for the broadband light curve. The results of these fits are reported in 289 Extended Data Table 3 and the inferred wavelength-dependent hot spot phases are shown in 290 Extended Data Fig. 10. A second suite of spectroscopic light curve fits was also performed with 291 the hot spot phase $\left(c_{2}\right)$ and higher-order phase curve terms $\left(c_{3}, c_{4}\right)$ held fixed to the maximum 292 likelihood values derived from the broadband light curve fit. The results of these fits are reported 293 in Extended Data Table 4. Derived heat redistribution factors $A_{F}$ (see above) for both suites of 294 spectroscopic light curve fits are reported in Extended Data Table 5. 
In all spectroscopic wavelength channels, the fits for which $c_{2}, c_{3}$, and $c_{4}$ were held fixed

296 have lower BIC values than the fits for which these parameters were allowed to vary freely

297 (Extended Data Table 5). For this reason, to derive the phase-resolved emission spectra of WASP-

$298121 \mathrm{~b}$ we adopt the light curve fits for which $c_{2}, c_{3}$, and $c_{4}$ were held fixed and show the

299 corresponding best-fit light curves in Extended Data Fig. 2. To extract the phase-resolved emission

300 spectra, the measured planetary emission for each spectroscopic light curve shown in Extended

301 Data Fig. 2 was binned into sixteen orbital phase bins, centred at phases $\phi=0.05,0.12,0.17,0.23$,

$3020.28,0.32,0.38,0.43,0.57,0.62,0.68,0.72,0.78,0.82,0.88$, and 0.95 , where $\phi=0$ coincides with

303 the primary transit mid-point and $\phi=0.5$ coincides with the secondary eclipse mid-point. Each

304 phase bin had an effective width of 1.5 hours with the exception of the bins centred at $\phi=0.05$ and

$305 \phi=0.95$, which had larger widths of 3 hours to compensate for the lower fluxes at those phases.

306 Uncertainties were calculated as the standard deviation of model residuals within each phase bin

307 added in quadrature to the standard deviation of in-eclipse model residuals (i.e. the uncertainty in

308 the stellar baseline flux level), following ref. 8. The planetary emission measurements obtained in

309 this way are reported in Extended Data Table 6 and plotted in Extended Data Figure 4. As

310 described in the main text, a dayside emission spectrum was also generated from the distribution

311 of light curve emission maxima generated during the fitting. This dayside spectrum is reported in

312 Extended Data Table 7 and, as shown in Extended Data Fig. 11, is in excellent agreement with the

313 spectrum obtained from the emission measured in the phase bin immediately preceding secondary

314 eclipse ingress. It also has a similar shape but a slightly higher overall level than the emission

315 measured in the phase bin immediately following eclipse egress. Both of these observations are

316 consistent with expectations, as the phase curve peak coincides with the phase bin immediately

317 preceding eclipse ingress (Fig. 1 and Extended Data Table 1). Furthermore, the secondary eclipse 
318 spectrum presented by ref. 3 also has a similar shape, but intermediate overall level, relative to 319 these spectra (Extended Data Fig. 11). Again, this is to be expected, as the secondary eclipse depths 320 of ref. 3 were measured relative to an out-of-eclipse baseline that was linear in $t$ and thus effectively 321 the average of the emission measured immediately before and after eclipse.

323 Retrieval analyses assuming chemical equilibrium

324 Atmospheric retrieval analyses were performed on the phase-resolved emission spectra using 325 ATMO, a one-dimensional radiative transfer code used to simulate substellar atmospheres. ${ }^{51-60}$ 326 ATMO solves the radiative transfer equation in plane-parallel geometry assuming hydrostatic and 327 radiative-convective equilibrium. The first step of the analysis was to perform a retrieval on the 328 dayside spectrum derived from the measured emission maxima in each spectroscopic wavelength 329 channel (Extended Data Table 7). For this retrieval, the pressure-temperature (PT) profile was 330 freely fit using the analytic profile of ref. 61 , with three free parameters: the infrared opacity $\left(\kappa_{I R}\right)$; 331 the ratio of the visible-to-infrared opacity $\left(\gamma=\kappa_{V} / \kappa_{I R}\right)$; and an irradiation efficiency factor $(\psi)$. The 332 atmosphere was assumed to be in chemical equilibrium, with the heavy element abundances (i.e. 333 metallicity) varied as a free parameter $([\mathrm{M} / \mathrm{H}])$. Chemical abundances were calculated using Gibbs

334 energy minimisation for 175 gaseous species, 9 ionic species, and 93 condensate species. ${ }^{56-59}$ 335 Rainout of condensed species consistent with the retrieved PT profiles was included, ${ }^{57}$ as was 336 thermal ionisation and dissociation. Opacities for the spectrally active species $\mathrm{H}_{2} \mathrm{O}, \mathrm{CO}_{2}, \mathrm{CO}, \mathrm{CH}_{4}$, $337 \mathrm{NH}_{3}, \mathrm{Na}, \mathrm{K}, \mathrm{Li}, \mathrm{Rb}, \mathrm{Cs}, \mathrm{TiO}, \mathrm{VO}, \mathrm{FeH}, \mathrm{PH}_{3}, \mathrm{H}_{2} \mathrm{~S}, \mathrm{HCN}, \mathrm{C}_{2} \mathrm{H}_{2}, \mathrm{SO}_{2}, \mathrm{Fe}$, and $\mathrm{H}^{-}$were included, 338 along with collision-induced absorption due to $\mathrm{H}_{2}-\mathrm{H}_{2}$ and $\mathrm{H}_{2}-\mathrm{He}$. Uniform priors were adopted 339 for all model parameters $\left(\kappa_{I R}, \gamma, \psi,[\mathrm{M} / \mathrm{H}]\right)$ and fitting was performed using nested sampling. ${ }^{62-64}$ 340 An ATLAS model ${ }^{65}$ was adopted for the stellar spectrum, assuming a stellar effective temperature 
$341 T_{\star}=6,500$ Kelvin, surface gravity $\log g_{\star}=4.0 \mathrm{~cm} \cdot \mathrm{s}^{-2}$, and radius $R_{\star}=1.458$ solar radii, based on the

342 values provided by ref. 9. The resulting posterior distributions are reported in Extended Data Table

3432 and the corresponding emission spectrum distribution is shown in Fig. 2a. The maximum

344 likelihood model has a $\chi^{2}$ value of 9.74 for 8 degrees of freedom (i.e. reduced $\chi_{v}^{2}=1.2$ ),

345 indicating a good fit to the data. Also shown in Fig. 3 are posterior distributions for the PT profile

346 (Fig. 3a), $\mathrm{H}_{2} \mathrm{O}$ abundance (Fig. 3b), and $\mathrm{H}^{-}$abundance (Fig. 3c), and the contribution function for

347 the maximum likelihood model (Fig. 3d). Note that the $\mathrm{H}_{2} \mathrm{O}$ and $\mathrm{H}^{-}$abundances were determined

348 from the chemical equilibrium abundances and were not fit directly as free parameters.

349 For the remaining phase-resolved emission spectra, varying fractions of the dayside and

350 nightside hemispheres are visible. Due to the strong contrast in effective temperature between each

351 hemisphere, the dayside and nightside spectra are expected to differ significantly. To

352 accommodate this, retrievals were performed using a method similar to the "2TP-Fixed"

353 framework described by ref. 25. Under this approach, denoted here as " $2 \mathrm{x}$ PT", the combined

354 emission received from the planet $\Phi$ at each orbital phase was assumed to be described by:

$355 \quad \Phi=\eta_{d} \Phi_{d}+\left(1-\eta_{d}\right) \Phi_{n}$

356 where $\eta_{d}$ is the fractional area of the visible dayside hemisphere, $\Phi_{d}$ is the planetary emission 357 from the dayside hemisphere, and $\Phi_{n}$ is the planetary emission from the nightside hemisphere. The 358 fractional area of the visible dayside hemisphere $\eta_{d}$ is given by:

$$
\eta_{d}=\left[1-\cos \left(2 \pi \phi+\pi-c_{2}\right)\right] / 2=\left[1+\cos \left(2 \pi \phi-c_{2}\right)\right] / 2
$$

360 This is a slight variation of Equation A2 in ref. 25, with $c_{2}$ corresponding to the phase of maximum 361 brightness and set to the value obtained from the broadband light curve fit (Extended Data Table 362 1). Given that the data are sensitive to thermal emission, rather than reflected light, the inclusion 363 of the $c_{2}$ offset accounts for the overall advection of gas prior to re-emission. The dayside emission 
$364 \Phi_{d}$ was also held fixed to the maximum likelihood model described above and shown in Fig. 2a.

365 This was done because the dayside spectrum derived from the emission maxima has a relatively 366 high signal-to-noise and retrieving for both the dayside and nightside contributions at each orbital 367 phase was not justified given the limited number of data points (i.e. twelve spectroscopic 368 channels). The metallicity was also assumed to be the same for both hemispheres and held fixed 369 to $[\mathrm{M} / \mathrm{H}]=0.7$ (i.e. $5 \mathrm{x}$ solar), close to the median value derived from the dayside spectrum 370 (Extended Data Table 2). With the metallicity and dayside emission held fixed, this left the three 371 PT profile parameters $\left(\kappa_{I R}, \gamma, \psi\right)$ for the nightside hemisphere as the remaining free parameters. As 372 for the initial retrieval for the dayside spectrum described above, fitting was again performed using 373 nested sampling, with uniform priors for the PT profile parameters. The nightside PT profiles were 374 retrieved in this way for phases $\phi=0.05,0.12,0.17,0.23,0.28,0.72,0.78,0.82,0.88$, and 0.95 . 375 Useful constraints could not be obtained by fitting for the nightside PT profiles at the remaining 376 phases (i.e. $\phi=0.32,0.38,0.43,0.57,0.62,0.68$ ), as the nightside emission $\Phi_{n}$ comprised a 377 relatively insignificant fraction of the total planetary emission. For these latter phases, $\Phi_{n}$ was 378 instead held fixed to the maximum likelihood model obtained for the $\phi=0.95$ retrieval (Fig. 2b), 379 as the nightside emission had the highest signal-to-noise at this phase, and the PT profile was 380 instead allowed to vary for the dayside emission component $\Phi_{d}$. Resulting phase-resolved 381 emission spectra are shown for all phases in Extended Data Fig. 4. The corresponding PT profiles, $382 \mathrm{H}_{2} \mathrm{O}$ abundances, and $\mathrm{H}^{-}$abundances are shown in Extended Data Figs 5, 6, and 7, respectively. 383 The $\chi^{2}$ fit statistics are reported in Extended Data Table 8, with a mean reduced $\chi_{v}^{2}=1.1$ and 384 median reduced $\chi_{v}^{2}=1.2$ achieved across the sixteen phase bins. One final retrieval was 385 performed for phase $\phi=0.95$, the same as before (i.e. dayside contribution held fixed and PT 386 parameters allowed to vary) but with the metallicity also allowed to vary as a free parameter. The 
387 results of this retrieval are given in Extended Data Table 2 and the inferred metallicity

$388\left([\mathrm{M} / \mathrm{H}]=0.66_{-1.02}^{+0.70}\right)$ is found to be consistent with the $[\mathrm{M} / \mathrm{H}]=0.7$ (i.e. $5 \mathrm{x}$ solar) value assumed for

389 the fiducial retrievals, providing a useful validation of the latter.

390 To assess the significance of the nightside emission detections, the $\chi^{2}$ and BIC values were

391 computed under the assumption of zero nightside emission (i.e. $\Phi_{n}=0$ ) at the ten phases for which

392 the " $2 \mathrm{x}$ PT" retrievals were performed for the nightside hemisphere. As reported in Extended Data

393 Table 9, the BIC values of the "2x PT" retrievals are lower than those of the retrievals assuming

$394 \Phi_{n}=0$ for seven of the ten phases considered. At three of the individual phases $(\phi=0.23,0.88$,

395 0.95) the Bayes factors are $>100$, corresponding to decisive preference for the " $2 \mathrm{x}$ PT" models

396 over the $\Phi_{n}=0$ null hypothesis. ${ }^{66,67}$ The strongest preference for the " $2 \mathrm{x}$ PT" retrieval is seen at

397 phase $\phi=0.95$, for which the null hypothesis is disfavoured by a Bayes factor of $7.1 \times 10^{10}$. This

398 translates to a preference for the " $2 \mathrm{x}$ PT" model over the $\Phi_{n}=0$ null hypothesis at a significance

399 in excess of $5 \sigma$ under the frequentist paradigm, following the conversion provided by ref. 68 . If all

400 ten phases are considered together as an ensemble, the BIC is 221.3 for the " $2 \mathrm{x}$ PT" retrievals and

401380.1 for the $\Phi_{n}=0$ null hypothesis (last row of Extended Data Table 9), amounting to an overall

402 rejection of the null hypothesis with a Bayes factor of $3 \times 10^{34}$. The unambiguous preference for 403 the " $2 \mathrm{x}$ PT" models over the $\Phi_{n}=0$ null hypothesis at multiple phases implies that emission from

404 the nightside hemisphere is distinct from the dayside contribution and detected at high confidence 405 in the data.

406

As an additional check, simple blackbody spectra were fit to each phase-resolved emission

407 spectrum. For these fits, the effective planetary temperature was the only free parameter. The 408 maximum likelihood spectra are plotted in Extended Data Figure 4 and the results are reported in 409 Extended Data Table 8. These blackbody fits had a mean reduced $\chi_{v}^{2}=2.1$ and median reduced 
$410 \chi_{v}^{2}=2.0$ across the sixteen phase bins, which is significantly poorer than those obtained for the 411 "2x PT" fits (Extended Data Table 8). However, the brightness temperatures derived from the 412 blackbody fits for the dayside and nightside hemispheres allow simple estimates to be made for 413 the planetary Bond albedo $\left(A_{\mathrm{B}}\right)$ and heat redistribution efficiency $(\varepsilon)$ following the method of ref.

414 69. The results of this analysis are shown in Extended Data Fig. 12a. As can be seen in Extended 415 Data Fig. 12b, the Bond albedo derived for WASP-121b $\left(A_{\mathrm{B}}=0.14 \pm 0.08\right)$ is consistent with 416 values reported for other hot Jupiters that have similar irradiation temperatures $\left(T_{0}=T_{\star} \sqrt{R_{\star} / a}=\right.$ $4173320 \pm 72 \mathrm{~K}$ for WASP-121b). However, the derived value for the heat redistribution efficiency $418(\varepsilon=0.29 \pm 0.02$ for WASP-121b) is notably higher than for two of those shown in Extended Data 419 Fig. 12c with similar irradiation temperatures: namely, WASP-18b $\left(T_{0}=3412 \pm 49, \varepsilon=\right.$ $\left.4200.01_{-0.01}^{+0.07}\right)^{70}$ and KELT-1b $\left(T_{0}=3391 \pm 29, \varepsilon=0.06_{-0.02}^{+0.03}\right){ }^{70}$ These measurements hint at a 421 diversity of circulation regimes among highly-irradiated substellar objects.

422

\section{Retrieval analyses with unconstrained chemistry}

424 A second suite of retrieval analyses was performed using the NEMESIS radiative transfer and

425 retrieval model. ${ }^{26,27,71-74}$ NEMESIS couples a parametric, one-dimensional radiative transfer 426 simulation to the PyMultiNest algorithm ${ }^{75}$ which uses nested sampling to explore the model 427 parameter space. ${ }^{60-62}$ The correlated-k approximation ${ }^{76}$ is used to pre-tabulate gas absorption data. There were a number of important differences between the approaches used by ATMO and 429 NEMESIS to model the WASP-121b atmosphere. First, the NEMESIS retrievals only fit for the 430 abundances of $\mathrm{H}_{2} \mathrm{O}$ and $\mathrm{H}^{-}$; the two other main species expected to be spectrally significant at the 431 wavelengths probed by the data are $\mathrm{VO}$ and $\mathrm{FeH}$ (Extended Data Fig. 3), which were included 432 with a constant mole fraction fixed to the values inferred by ref. 20. Second, the abundances of 
$433 \mathrm{H}_{2} \mathrm{O}$ and $\mathrm{H}^{-}$were allowed to vary freely at each orbital phase, without the requirement of satisfying

434 chemical equilibrium. Third, following ref. 10, a simple analytic treatment was adopted to account

435 for thermal dissociation of $\mathrm{H}_{2} \mathrm{O}$ at phases for which the dayside was retrieved (i.e. $\phi=0.32,0.38$,

$4360.43,0.57,0.62,0.68)$. Specifically, the following parameterisation for the $\mathrm{H}_{2} \mathrm{O}$ mole fraction $(X)$

437 as a function of pressure $(P)$ was adopted:

438

$$
X(P)= \begin{cases}X_{\text {deep }} & \text { for } P \geq P_{\text {knee }} \\ X_{\text {deep }}\left(P / P_{\text {knee }}\right)^{\alpha} & \text { for } P<P_{\text {knee }}\end{cases}
$$

439 where the deep atmosphere $\mathrm{H}_{2} \mathrm{O}$ mole fraction $\left(X_{\text {deep }}\right)$, knee pressure $\left(P_{\text {knee }}\right)$, and power law index

$440(\alpha)$ were fitted as free parameters. As in ref. $20, \mathrm{H}^{-}$was assumed to be well-mixed throughout the

441 atmosphere, with constant mole fraction retrieved as an additional parameter. The remaining

442 atmosphere was assumed to be composed of $\mathrm{H}_{2}$ and $\mathrm{He}$ in a 9:1 ratio.

443 The $\mathrm{H}_{2} \mathrm{O}, \mathrm{VO}$, and $\mathrm{FeH}$ k-tables were computed according to ref. 77, using the data

444 presented in refs. 78, 79, and 80, respectively. The $\mathrm{H}^{-}$bound-free and free-free opacities were

445 calculated according to ref. 81. Also included were $\mathrm{e}^{-}$and $\mathrm{H}$, both assumed to be well-mixed with

446 abundances fixed to the deep atmosphere $\mathrm{H}^{-}$abundance. The latter was justified because the

447 abundances of $\mathrm{e}^{-}$and $\mathrm{H}$ do not affect the observed emission spectrum provided that the abundances

448 are sufficiently high to allow significant interaction with the $\mathrm{H}^{-}$ions. Collision-induced absorption

449 due to $\mathrm{H}_{2}-\mathrm{H}_{2}$ and $\mathrm{H}_{2}-\mathrm{He}$ was also included. ${ }^{82-86}$ A parameterisation identical to that used by the

450 ATMO retrievals was adopted for the PT profile. At each orbital phase, this gave a final model

451 with seven free parameters: three parameters for the PT profile $\left(\kappa_{I R}, \gamma, \psi\right)$; the $\mathrm{H}^{-}$mole fraction;

452 and the three parameters defined above for the pressure-dependent $\mathrm{H}_{2} \mathrm{O}$ mole fraction $\left(X_{\text {deep }}\right.$, $\left.453 P_{\text {knee }}, \alpha\right)$. 
Results for the fiducial dayside and nightside hemisphere PT profiles, $\mathrm{H}_{2} \mathrm{O}$ abundances,

455 and $\mathrm{H}^{-}$abundances are compared to those obtained by the ATMO retrievals in Extended Data Fig.

456 8. Maximum likelihood emission spectra are shown for all orbital phases in Extended Data Fig. 4,

457 with the PT profiles, $\mathrm{H}_{2} \mathrm{O}$ abundances, and $\mathrm{H}^{-}$abundances that were retrieved separately for each

458 phase shown in Extended Data Figs 5, 6, and 7, respectively. Overall, the PT profiles inferred by

459 NEMESIS for the nightside phases are in good agreement with those inferred by ATMO. The

460 agreement is reasonable, but not as good, for the dayside phases. The latter is likely due to the

461 challenge of accounting for thermal dissociation and ionisation using the parameterised approach

462 described above for the free chemistry NEMESIS retrievals. The NEMESIS retrievals at dayside

463 phases do not succeed in accounting for the thermal dissociation of $\mathrm{H}_{2} \mathrm{O}$ (Extended Data Fig. 8b).

464 Instead, to account for the muted $\mathrm{H}_{2} \mathrm{O}$ spectral band, the NEMESIS retrieval favours a higher $\mathrm{H}^{-}$

465 abundance compared to ATMO (Extended Data Fig. 8c), which raises the opacity at wavelengths

466 shortward of $1.3 \mu \mathrm{m}$ in particular (Extended Data Fig. 3). The overall raised opacity produces an

467 extended wing in the contribution function towards lower pressures (Extended Data Fig. 8d), in

468 turn favouring a thermal inversion at lower pressures than ATMO (Extended Data Fig. 8a). This

469 also explains why NEMESIS infers thermal inversions at lower pressures than ATMO for phases

$470 \phi=0.43,0.57$, and 0.62 (Extended Data Fig. 5).

471 However, the broad agreement between the NEMESIS and ATMO results is reassuring,

472 given the different methodologies adopted. The NEMESIS $\chi^{2}$ fit statistics are reported alongside 473 those for ATMO in Extended Data Table 8. For NEMESIS, the mean reduced $\chi_{v}^{2}=2.43$ and the

474 median reduced $\chi_{v}^{2}=2.34$, which are significantly higher than the equivalent fit quality metrics 475 achieved by ATMO. This is primarily a consequence of the larger number of parameters required 476 for the NEMESIS retrievals (i.e. seven for NEMESIS versus three for ATMO), although the 
477 absolute $\chi^{2}$ values are also higher, indicating that the NEMESIS models do not replicate the data

478 as well as the ATMO models overall. The latter is due to the failure of NEMESIS to adequately

479 treat the thermal dissociation of $\mathrm{H}_{2} \mathrm{O}$ for the dayside spectra, as noted above. For these reasons,

480 along with the physically-motivated enforcement of chemical equilibrium, we present the ATMO

481 retrievals as our primary analysis (Figs 2 and 3).

\section{General circulation models}

484 A three-dimensional general circulation model (GCM) simulation was performed for the 485 atmosphere of WASP-121b using the Substellar and Planetary Radiation and Circulation (SPARC)

486 model. ${ }^{87-95}$ The model couples the MITgcm dynamical core, ${ }^{96}$ a finite-volume code that solves the 487 three-dimensional primitive equations on a staggered Arakawa $\mathrm{C}$ grid, ${ }^{97}$ with a plane-parallel, two488 stream version of a multi-stream radiation code developed for planetary atmospheres. ${ }^{98}$ Opacities 489 are calculated using the correlated- $k \operatorname{method}^{99}$ assuming local thermodynamic and chemical 490 equilibrium for each PT point, using the solar photospheric elemental abundances of ref. 100. In 491 particular, the model includes opacity due to important absorbers such as $\mathrm{H}_{2} \mathrm{O}, \mathrm{H}^{-}, \mathrm{CO}$, TiO, and 492 VO, but does not yet include atomic metals such as Fe and Mg. The coupling of the dynamical 493 core and radiative transfer scheme allow for the self-consistent calculation of the heating and 494 cooling rates of the atmosphere.

The SPARC GCM for WASP-121b has a horizontal resolution of C32 (128×64 in longitude

496 and latitude, respectively) and a vertical resolution of 45 pressure levels evenly spaced in log 497 pressure, that extend from a mean pressure of 1,000 bar at the bottom to $200 \mu$ bar at the top. The 498 model was integrated for 80 Earth days ( $\sim 00$ planetary orbits). A global map of the temperature 499 and wind speeds at a pressure of 10 mbar (a pressure within the range of altitudes probed at the 
500 WFC3 wavelengths, e.g. Fig. 3d) is shown in Extended Data Fig. 13. The map shows 501 predominantly eastward flow at the equator and nightside vortices, with dayside temperatures 502 exceeding 3,000 Kelvin and nightside temperatures dropping to $\sim 1,000$ Kelvin. Synthetic phase 503 curves were generated from the GCM following refs 24 and 101, and are shown in Fig. 1 and 504 Extended Data Fig. 2. Predicted emission spectra are shown in Fig. 2 and Extended Data Fig. 4. 505 Also shown in the latter figures are predicted emission spectra from the independent GCM 506 simulations of ref. 10, which were performed for atmospheric metallicities of $1 \mathrm{x}$ and $5 \mathrm{x}$ solar.

507 Two important caveats are worth highlighting, which apply to the GCM simulation 508 presented here, as well as those published by ref. 10. First, the lack of opacity due to metals such 509 as Fe and Mg could be significant, as separate modelling has shown that these metals can play 510 major roles in determining the outgoing emission for ultrahot Jupiters such as WASP-121b. ${ }^{11}$ 511 Second, the atmosphere of WASP-121b was assumed to be cloud-free to simplify the modelling. 512 However, clouds could potentially play a significant role in the atmospheric radiative transfer for 513 WASP-121b, particularly on the nightside hemisphere and in the terminator region where 514 temperatures are relatively low and likely conducive to the condensation of numerous species (Fig. $5153 b)$. 


\section{Data availability}

Raw HST data frames are publicly available online at the Mikulski Archive for Space Telescopes (MAST; https://archive.stsci.edu).

\section{Code availability}

The main analysis routines have been written by the authors in Python and are available on request. Other publicly available code that was used has been cited throughout the text.

\section{Methods References}

41. Stevenson, K. B., Fowler, J. Analysing Eight Years of Transiting Exoplanet Observations Using WFC3's Spatial Scan Monitor. Space Telescope Science Institute WFC3 Instrument Science Report. 2019-12 (2019).

42. Evans, T. M. et al. Detection of H- and Evidence for TiO/VO in an Ultra-hot Exoplanet Atmosphere. Astrophys. $J .822,(2016)$.

43. Evans, T. M. et al. An ultrahot gas-giant exoplanet with a stratosphere. Nature. 548, (2017).

44. Mikal-Evans et al. An emission spectrum for WASP-121b measured across the $0.8-1.1 \mu \mathrm{m}$ wavelength range using the Hubble Space Telescope. Mon. Not. R. Astron. Soc. 488, (2019).

45. de Wit, J. et al. Atmospheric reconnaissance of the habitable-zone Earth-sized planets orbiting TRAPPIST-1. Nature Astron. 2, (2018).

46. Kreidberg, L. batman: BAsic Transit Model cAlculatioN in Python. Publ. Astron. Soc. Pac. 127, (2015).

47. Foreman-Mackey, D., Hogg, D. W., Lang, D., Goodman, J. emcee: The MCMC Hammer. Publ. Astron. Soc. Pac. 125, (2013).

48. Parmentier, V. \& Crossfield, I. J. M. Exoplanet phase curves: observations and theory. Handbook of Exoplanets, ed. Deeg, H. J. \& Belmonte, J. A. Springer Publishing, Cham (2018).

49. Louden, T., Kreidberg, L. SPIDERMAN: an open-source code to model phase curves and secondary eclipses. Mon. Not. R. Astron. Soc. 477, (2018). 
50. Deming, D. et al. Infrared Transmission Spectroscopy of the Exoplanets HD 209458b and XO-1b Using the Wide Field Camera-3 on the Hubble Space Telescope. Astrophys. J. 774, (2013).

51. Tremblin, P. et al. Fingering Convection and Cloudless Models for Cool Brown Dwarf Atmospheres. Astrophys. J. 804, (2015).

52. Tremblin, P. et al. Cloudless Atmospheres for L/T Dwarfs and Extrasolar Giant Planets. Astrophys. J. 817, (2016).

53. Tremblin, P. et al. Advection of Potential Temperature in the Atmosphere of Irradiated Exoplanets: A Robust Mechanism to Explain Radius Inflation. Astrophys. J. 841, (2017).

54. Tremblin, P. et al. Cloudless Atmospheres for Young Low-gravity Substellar Objects. Astrophys. J. 850, (2017).

55. Amundsen, D. S. et al. Accuracy tests of radiation schemes used in hot Jupiter global circulation models. Astron. Astrophys. 564, (2014).

56. Drummond, B. et al. The effects of consistent chemical kinetics calculations on the pressure-temperature profiles and emission spectra of hot Jupiters. Astron. Astrophys. 594, (2016).

57. Goyal, J. M. et al. A library of ATMO forward model transmission spectra for hot Jupiter exoplanets. Mon. Not. R. Astron. Soc. 474, (2018).

58. Goyal, J. M. et al. Fully scalable forward model grid of exoplanet transmission spectra. Mon. Not. R. Astron. Soc. 482, (2019).

59. Goyal, J. M. et al. A library of self-consistent simulated exoplanet atmospheres. Mon. Not. R. Astron. Soc. 498, $4680(2020)$.

60. Phillips, M. W. et al. A new set of atmosphere and evolution models for cool T-Y brown dwarfs and giant exoplanets. Astron. Astrophys. 637, (2020).

61. Guillot, T. On the radiative equilibrium of irradiated planetary atmospheres. Astron. Astrophys. 520, A27 (2010).

62. Feroz, F., Hobson, M. P. Multimodal nested sampling: an efficient and robust alternative to Markov Chain Monte Carlo methods for astronomical data analyses. Mon. Not. R. Astron. Soc. 384, (2008).

63. Feroz, F., Hobson, M. P., Bridges, M. MULTINEST: an efficient and robust Bayesian inference tool for cosmology and particle physics. Mon. Not. R. Astron. Soc. 398, (2009).

64. Feroz, F., Hobson, M. P., Cameron, E., Pettitt, A. N. Importance Nested Sampling and the MultiNest Algorithm. Open J. Astrophys. 2, (2019). 
65. Castelli, F. \& Kurucz, R. L. New grids of ATLAS9 model atmospheres. Preprint at https://arxiv.org/abs/astro$\mathrm{ph} / 0405087$ (2004).

66. Kass, R. E. \& Raftery, E. Bayes factors. J. Am. Stat. Assoc. 90, 773 (1995).

67. Jeffreys, H. The Theory of Probability (3 ${ }^{\text {rd }}$ ed.). Oxford Univ. Press, Oxford (1998).

68. Trotta, R. Bayes in the sky: Bayesian inference and model selection in cosmology. Contemp. Phys. 49, 71 (2008).

69. Cowan, N. B., Agol, E. The statistics of albedo and heat recirculation of hot exoplanets. Astrophys. J. 729,54 (2011).

70. Keating, D., Cowan, N. B., Dang, L. Uniformly hot nightside temperatures on short-period gas giants. Nat. Astro. 3, $1092(2019)$.

71. Perna, R., Menou, K., Rauscher, E. Magnetic Drag on Hot Jupiter Atmospheric Winds. Astrophys. J. 719, 1421 (2010).

72. Batygin, K., Stanley, S., Stevenson, D. J. Magnetically controlled circulation on hot extrasolar planets. Astrophys. J. 776, 53 (2013).

73. Irwin, P. G. J. et al. The NEMESIS planetary atmosphere radiative transfer and retrieval tool. J. Quant. Spectrosc. Radiat. Transf. 109, (2008).

74. Lee, J.-M., Fletcher, L. N., Irwin, P. G. J. Optimal estimation retrievals of the atmospheric structure and composition of HD 189733b from secondary eclipse spectroscopy. Mon. Not. R. Astron. Soc. 420, (2012).

75. Barstow, J. K., Aigrain, S., Irwin, P. G. J., Sing, D. K. A Consistent Retrieval Analysis of 10 Hot Jupiters Observed in Transmission. Astrophys. J. 834, (2017).

76. Krissansen-Totton, J., Garland, R., Irwin, P., Catling, D. C. Detectability of Biosignatures in Anoxic Atmospheres with the James Webb Space Telescope: A TRAPPIST-1e Case Study. Astron. J. 156, (2018).

77. Buchner, J. et al. X-ray spectral modelling of the AGN obscuring region in the CDFS: Bayesian model selection and catalogue. Astron. Astrophys. 564, (2014).

78. Lacis, A. A., Oinas, V. A description of the correlated-k distribution method for modelling nongray gaseous absorption, thermal emission, and multiple scattering in vertically inhomogeneous atmospheres. J. Geophys. Res. 96, 9027 (1991).

79. Chubb, K. L. et al. The ExoMolOP Database: Cross-sections and k-tables for Molecules of Interest in HighTemperature Exoplanet Atmospheres. Preprint at https://arxiv.org/abs/2009.00687 (2020). 
80. Polyansky, O. L. et al. ExoMol molecular line lists XXX: a complete high-accuracy line list for water. Mon. Not. R. Astron. Soc. 480, (2018).

81. McKemmish, L. K., Yurchenko, S. N., Tennyson, J. ExoMol line lists - XVIII. The high-temperature spectrum of VO. Mon. Not. R. Astron. Soc. 463, 771 (2016).

82. Wende, S., Reiners, A., Seifahrt, A., Bernath, P. F. CRIRES spectroscopy and empirical line-by-line identification of FeH molecular absorption in an M dwarf. Astron. Astrophys. 523, A58 (2010).

83. John, T. L. Continuous absorption by the negative hydrogen ion reconsidered. Astron. Astrophys. 193 , 189 (1988).

84. Borysow, A. Collision-induced absorption coefficients of $\mathrm{H} 2$ pairs at temperatures from $60 \mathrm{~K}$ to $1000 \mathrm{~K}$. Astron. Astrophys. 390, 779 (2002).

85. Borysow, A., Frommhold, L. Collision-induced Infrared Spectra of H 2-He Pairs at Temperatures from 18 to 7000 K. II. Overtone and Hot Bands. Astrophys. J. 341, (1989).

86. Borysow, A., Frommhold, L. A New Computation of the Infrared Absorption by H 2 Pairs in the Fundamental Band at Temperatures from 600 to 5000 K. Astrophys. J. 348, (1990).

87. Borysow, A., Frommhold, L., Moraldi, M. Collision-induced Infrared Spectra of H 2-He Pairs Involving 01 Vibrational Transitions and Temperatures from 18 to 7000 K. Astrophys. J. 336, (1989).

88. Borysow, A., Jorgensen, U. G., Fu, Y. High-temperature (1000-7000 K) collision-induced absorption of H"2 pairs computed from the first principles, with application to cool and dense stellar atmospheres. J. Quant. Spectrosc. Radiat. Transf. 68, (2001).

89. Showman, Adam P. et al. Atmospheric Circulation of Hot Jupiters: Coupled Radiative-Dynamical General Circulation Model Simulations of HD 189733b and HD 209458b. Astrophys. J. 699, (2009).

90. Showman, A. P., Fortney, J. J., Lewis, N. K., Shabram, M. Doppler Signatures of the Atmospheric Circulation on Hot Jupiters. Astrophys. J. 762, (2013).

91. Showman, A. P., Lewis, N. K., Fortney, J. J. 3D Atmospheric Circulation of Warm and Hot Jupiters. Astrophys. J. 801, (2015).

92. Kataria, T. et al. Three-dimensional Atmospheric Circulation of Hot Jupiters on Highly Eccentric Orbits. Astrophys. J. 767, (2013). 
93. Kataria, T., Showman, A. P., Fortney, J. J., Marley, M. S., Freedman, R. S. The Atmospheric Circulation of the Super Earth GJ 1214b: Dependence on Composition and Metallicity. Astrophys. J. 785, (2014).

94. Kataria, T. et al. The Atmospheric Circulation of the Hot Jupiter WASP-43b: Comparing Three-dimensional Models to Spectrophotometric Data. Astrophys. J. 801, (2015).

95. Kataria, T. et al. The Atmospheric Circulation of a Nine-hot-Jupiter Sample: Probing Circulation and Chemistry over a Wide Phase Space. Astrophys. J. 821, (2016).

96. Lewis, Nikole K. et al. Atmospheric Circulation of Eccentric Hot Neptune GJ436b. Astrophys. J. 720, (2010).

97. Lewis, N. K., Showman, A. P., Fortney, J. J., Knutson, H. A., Marley, M. S. Atmospheric Circulation of Eccentric Hot Jupiter HAT-P-2b. Astrophys. J. 795, (2014).

98. Adcroft, A., Campin, J.-M., Hill, C., Marshall, J. Implementation of an Atmosphere Ocean General Circulation Model on the Expanded Spherical Cube. Mon. Weather Rev. 132, 2845 (2004).

99. Arakawa, A., Lamb, V. R. Computational design of the basic dynamical processes of the UCLA general circulation model. Methods in Computational Physics: Advances in Research and Applications. 17, 173 (1977).

100. Marley, M. S., McKay, C. P. Thermal Structure of Uranus' Atmosphere. Icarus. 138, 268 (1999).

101. Goody, R. M., Yung, Y. L. Atmospheric radiation: Theoretical basis. Oxford University Press, New York/Oxford (1989).

102. Lodders, K. Solar System Abundances and Condensation Temperatures of the Elements. Astrophys. J. 591,1220 (2003).

103. Fortney, J. J., Cooper, C. S., Showman, A. P., Marley, M. S., Freedman, R. S. The Influence of Atmospheric Dynamics on the Infrared Spectra and Light Curves of Hot Jupiters. Astrophys. J. 652, (2006). 


\section{Acknowledgements}

Support for HST program GO-15134 was provided by NASA through a grant from the Space

Telescope Science Institute, which is operated by the Association of Universities for Research in Astronomy, Inc., under NASA contract NAS 5-26555. N.M. is supported by a UKRI Future Leaders Fellowship: MR/T040866/1, a Science and Technology Facilities Council Consolidated Grant (ST/R000395/1) and the Leverhulme Trust through a research project grant (RPG-2020-82).

\section{Author Contributions}

T.M.E. co-led the observing program, analysed the HST data, and wrote the manuscript. D.K.S., J.K.B, and J.T. performed the retrieval analyses. T.K. co-led the observing program and ran the GCM simulation. J.G. and N.L. processed the GCM results. T. D. assisted with the light curve analysis. All authors discussed the results and provided feedback on the manuscript.

\section{Competing Interest Declaration}

The authors declare no competing interests. 


\section{EXTENDED DATA}

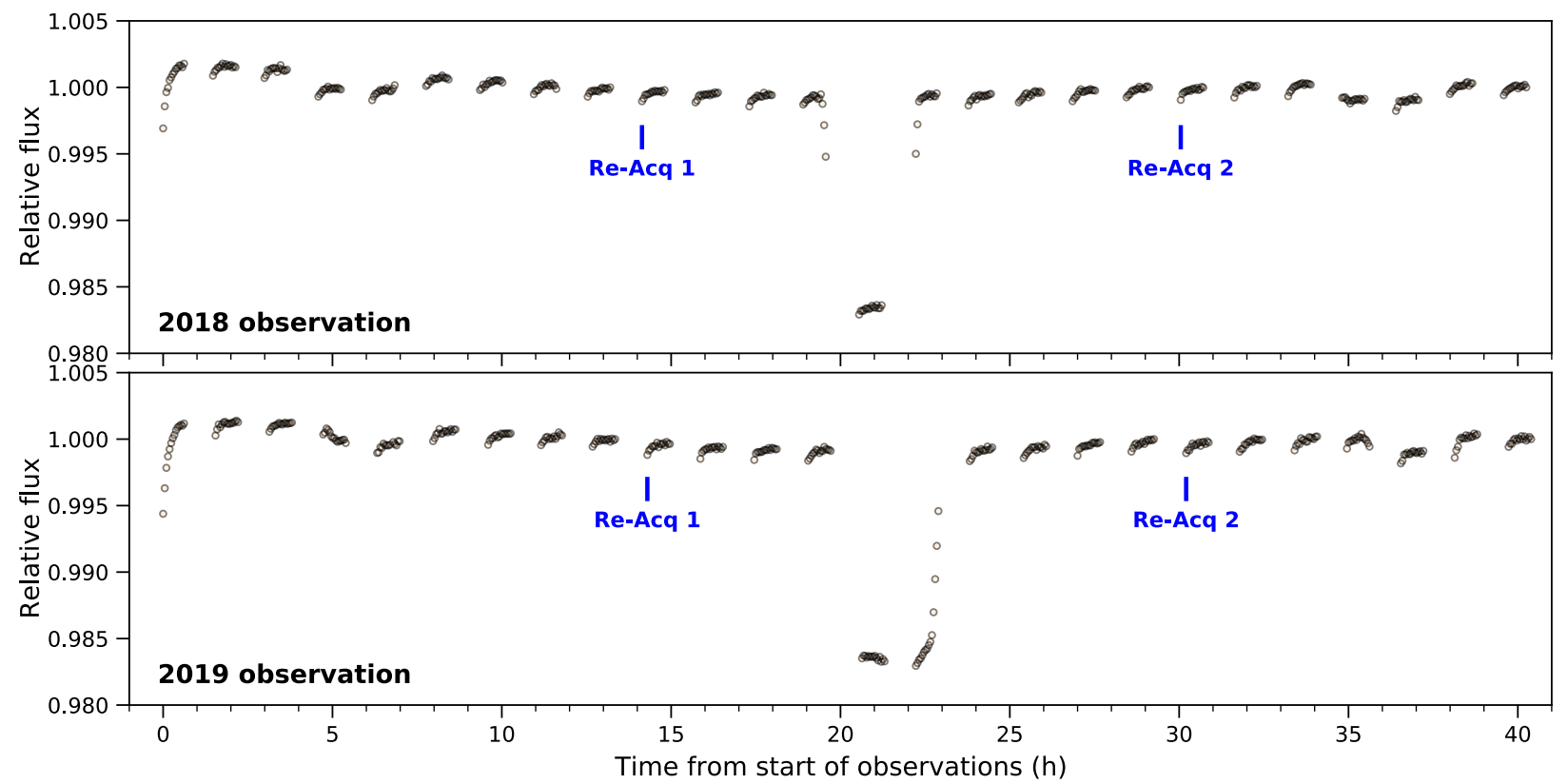

Extended Data Fig. 1. Raw broadband light curves for WASP-121b. WFC3 observations made in 2018 and 2019.

Gaps in the time series are due to the target disappearing from view for approximately half of each HST orbit. Two secondary eclipses and the primary transit are visible by eye. The data are affected by detector systematics that result in a ramp-up of flux registered during each HST orbit. Guide star re-acquisitions were performed at the beginning of the 10th and 20th HST orbits for both observations. 

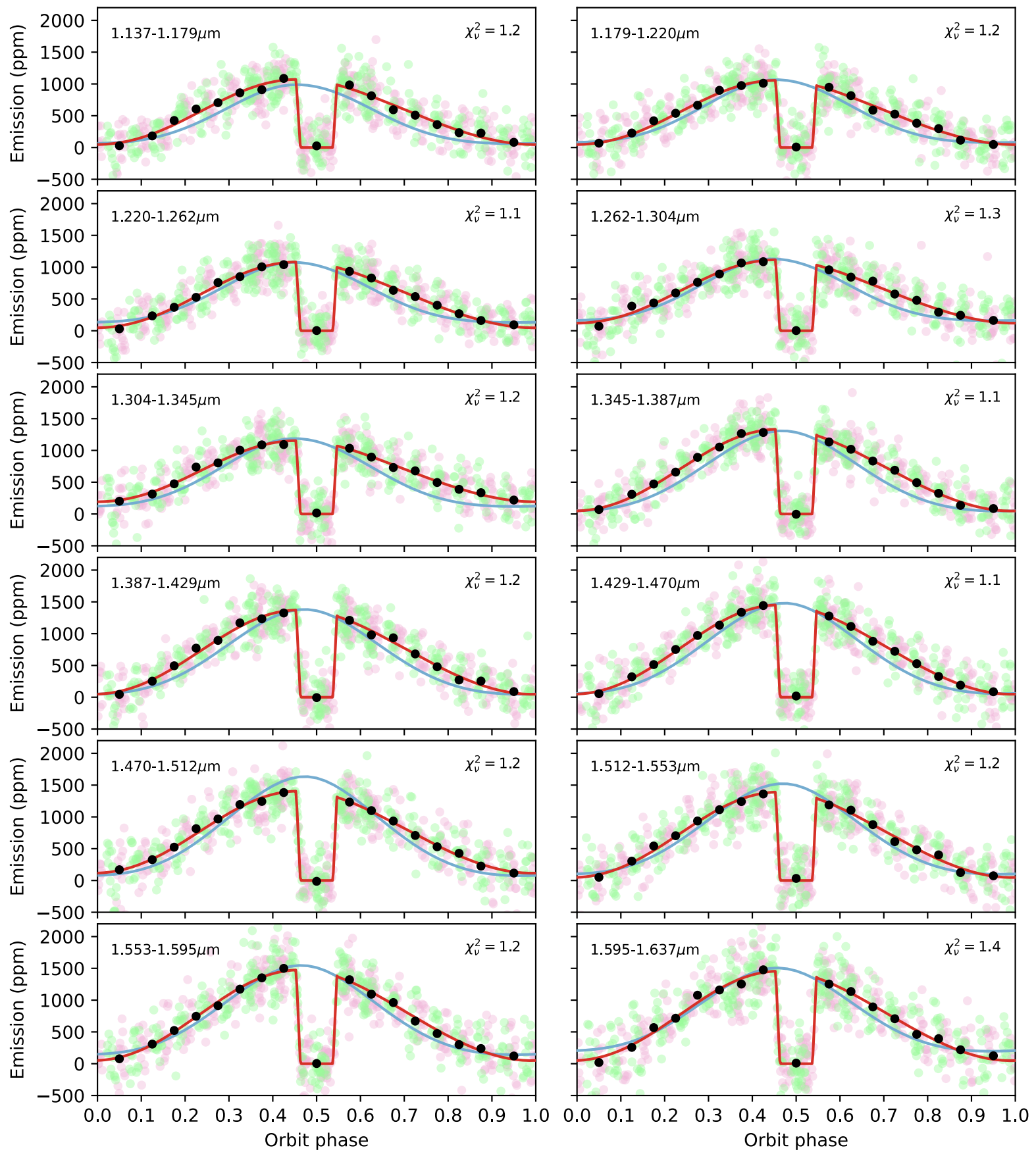

Extended Data Fig. 2. Spectroscopic phase curves for WASP-121b. Systematics-corrected spectroscopic phase curves measured with WFC3. Pink circles show 2018 data and green circles show 2019 data. Black circles show the combined dataset binned in phase, with marker sizes approximately corresponding to the measurement uncertainties. Phase bins are the same as those used for generating the phase-resolved emission spectra, plus an additional in-eclipse bin. Red lines show the maximum likelihood second-order sinusoidal fits, with corresponding reduced $\chi_{v}^{2}$ values listed in the upper right corner of each axis. Blue lines show predictions of the 3D GCM run for the present study. 


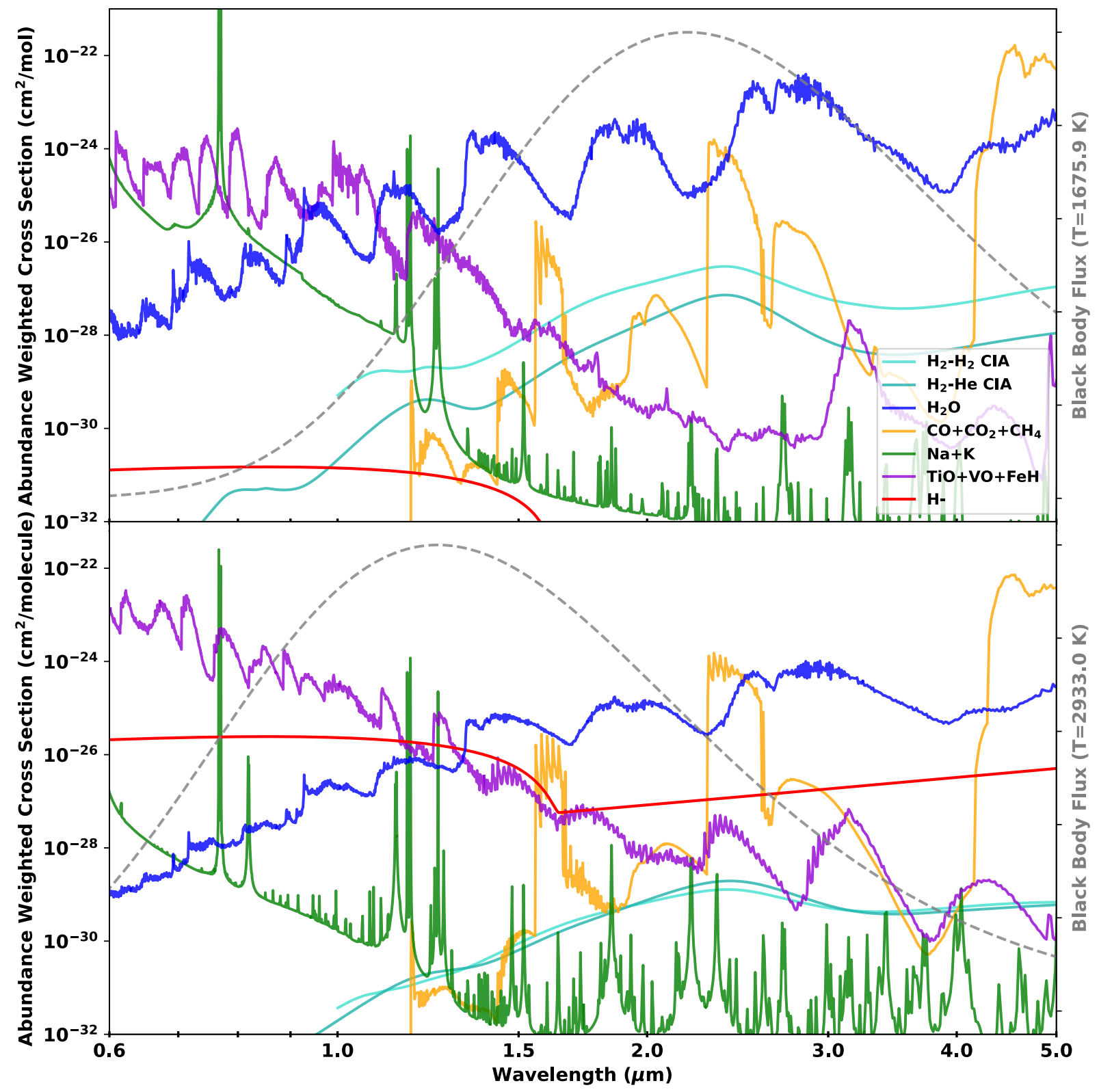

Extended Data Fig. 3. Spectrally active gases on the nightside (top panel) and dayside (bottom panel) hemispheres. Solid lines show absorption cross-sections for spectrally active species weighted by their mole fractions at pressures just below the contribution function peaks, as inferred by the ATMO " $2 \mathrm{x}$ PT" retrieval analyses. Dashed grey lines show blackbody emission curves for the retrieved temperatures at the same pressure levels. 


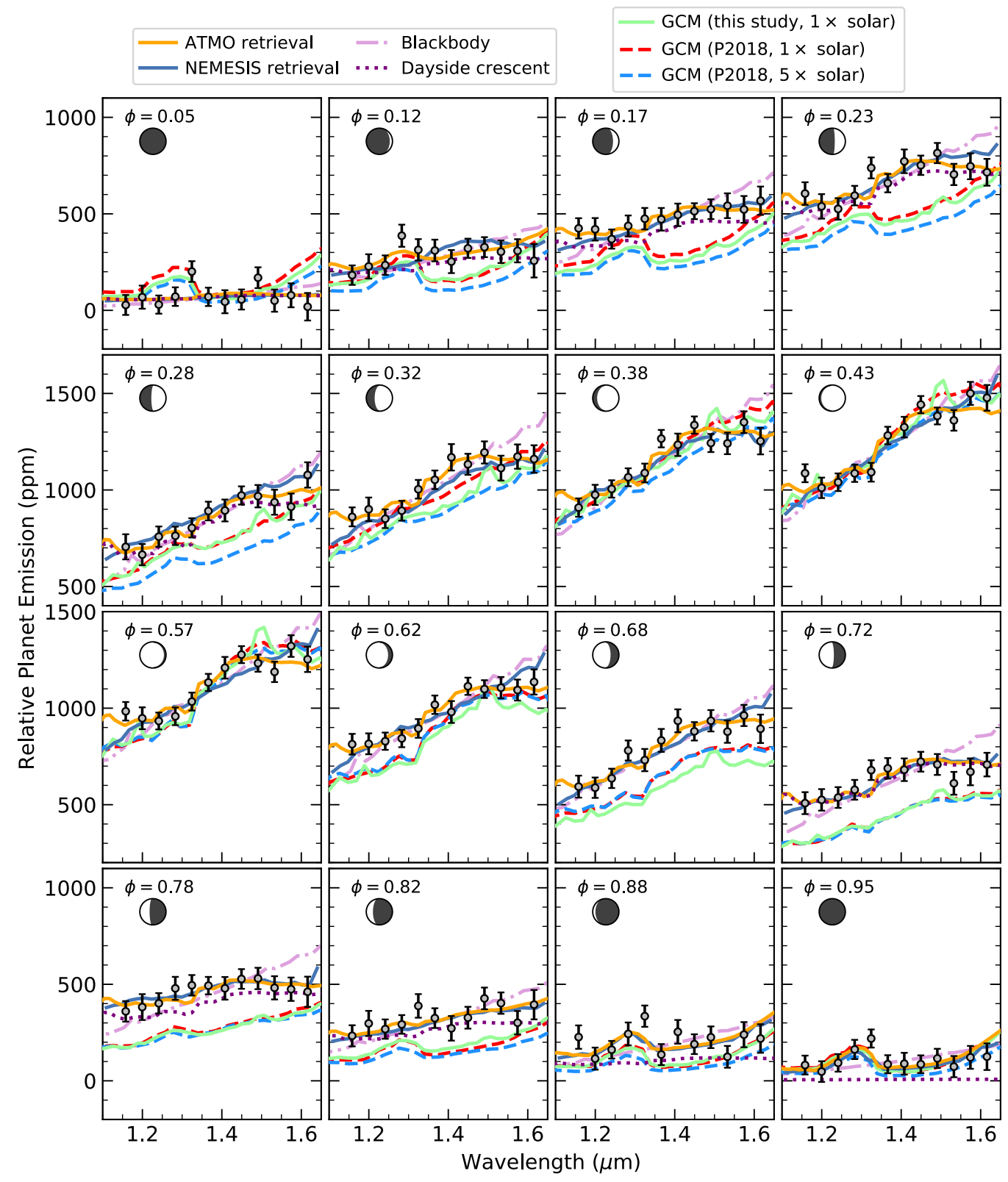

Extended Data Fig. 4. Emission spectra at different orbital phases for WASP-121b. Grey circles show measured planet emission as a function of wavelength with error bars indicating $1 \sigma$ measurement uncertainties. Solid orange and dark blue lines show the maximum likelihood spectra obtained from the ATMO "2x PT" retrievals and NEMESIS "2x PT" retrievals, respectively. Dotted purple lines show dayside contributions for the phases at which retrievals were performed for the nightside emission. Solid light green line shows the emission predicted by the 3D GCM run for the present study assuming 1x solar metallicity. Dashed red and blue lines show the emission predicted by the 3D GCM simulations of ref 10 assuming 1x and 5x solar metallicity, respectively. Dot-dashed purple lines show best-fit blackbody spectra. Circle symbols indicate the illuminated fraction of the visible hemisphere at each orbital phase. 


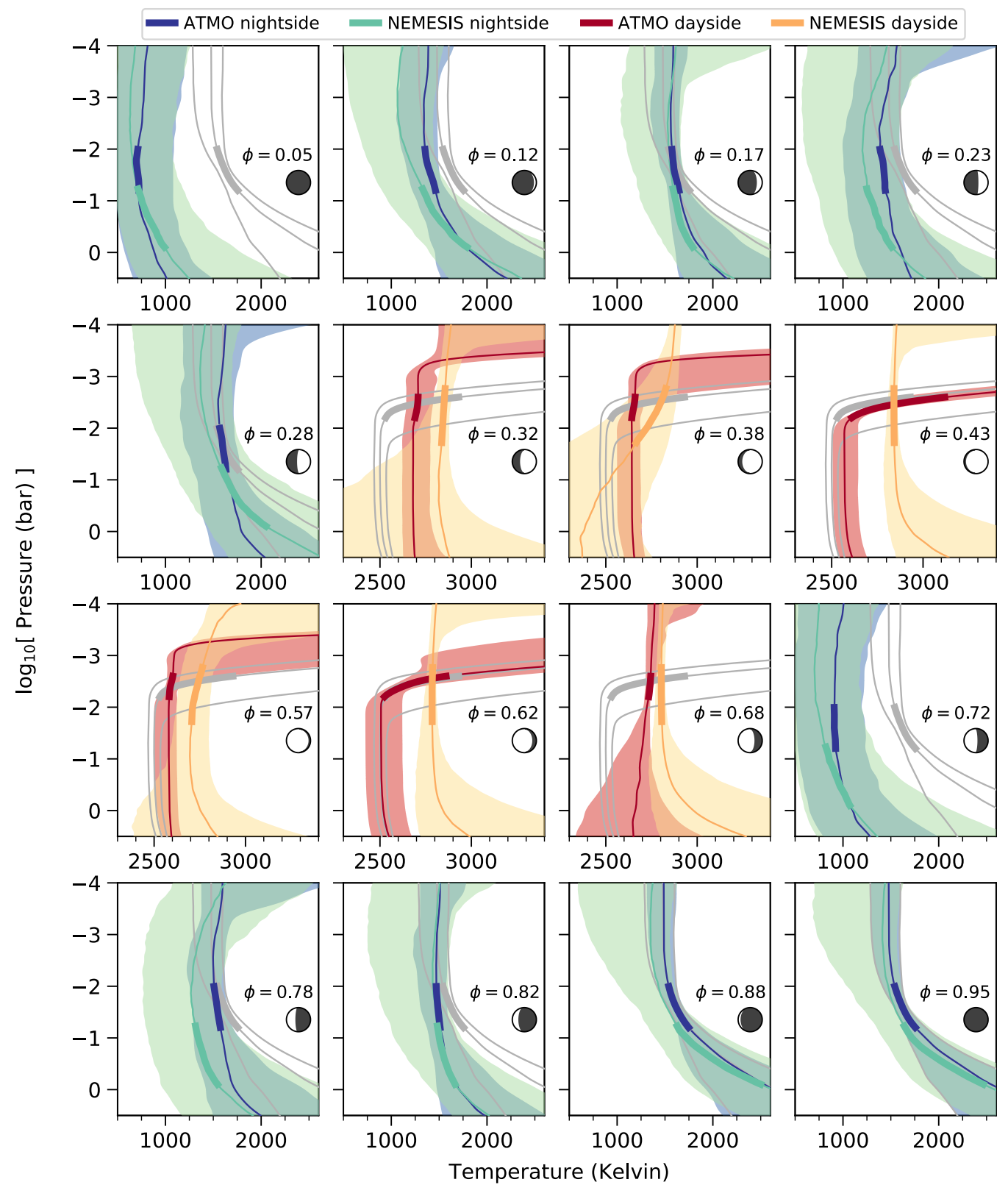

Extended Data Fig. 5. Pressure-temperature (PT) profiles retrieved for different orbital phases. Blue lines show the median PT profiles obtained from the ATMO " $2 \mathrm{x}$ PT" nightside retrievals, with blue shading the corresponding $1 \sigma$ credible ranges. Red lines and shading show the same for the ATMO "2x PT" dayside retrievals. Green and yellow lines and shading show the same for the NEMESIS " $2 \mathrm{x}$ PT" nightside and dayside retrievals, respectively. For the ATMO retrievals, thick lines correspond to the same pressures highlighted in Fig. 3, where the contribution function is greatest. For the NEMESIS retrievals, thick lines indicate the equivalent pressures of greatest contribution for those retrievals (Extended Data Fig. 8d). Grey lines reproduce the fiducial dayside and nightside PT distributions of Fig. 3. 


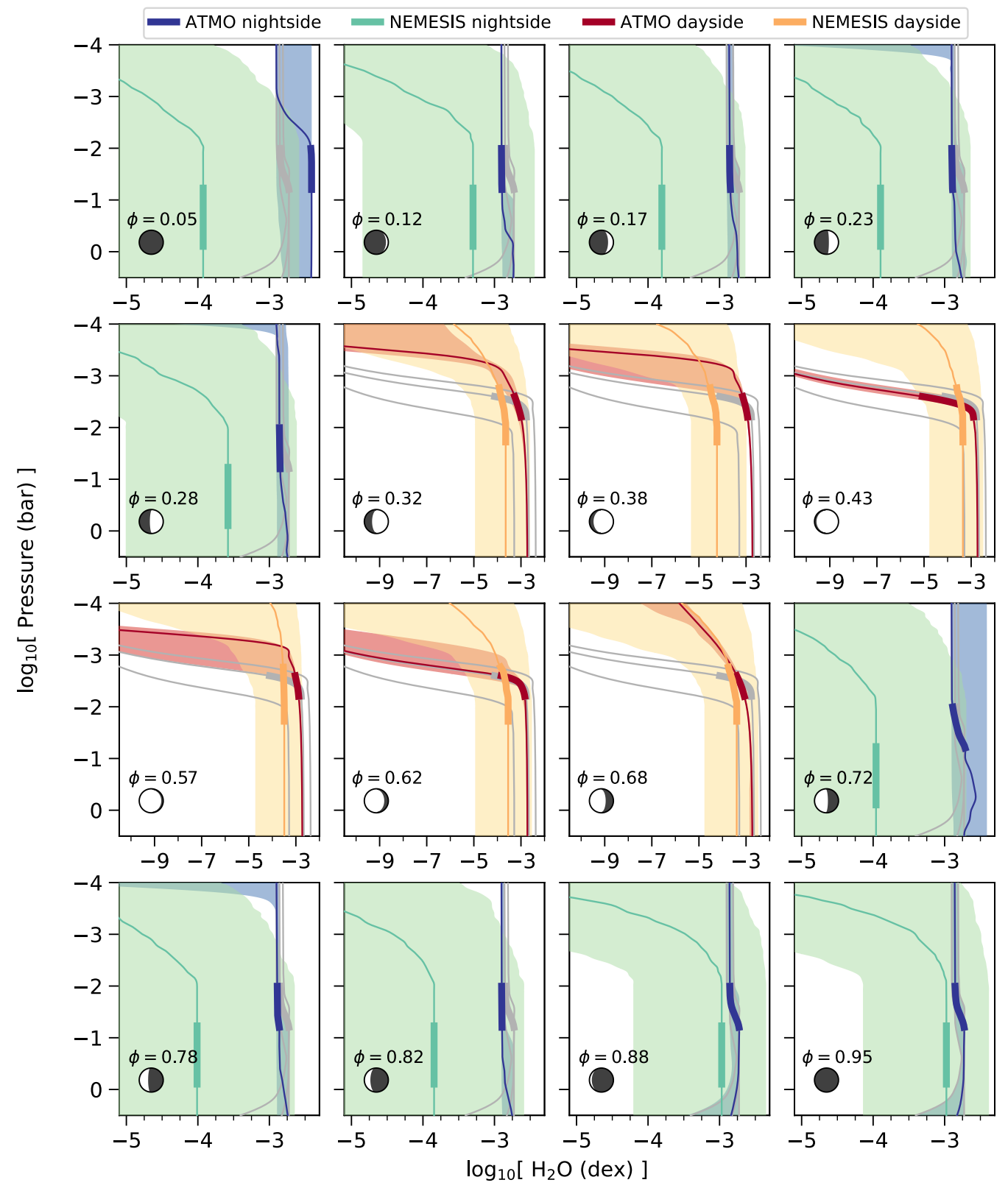

Extended Data Fig. 6. Retrieved pressure-dependent $\mathrm{H}_{2} \mathrm{O}$ abundances. Same format as Extended Data Figure 5, but showing posterior distributions for the retrieved $\mathrm{H}_{2} \mathrm{O}$ abundances. ATMO credible ranges are much narrower than those of NEMESIS, due to the metallicity being fixed for the ATMO retrievals whereas the $\mathrm{H}_{2} \mathrm{O}$ abundance was unconstrained for the NEMESIS retrievals. 


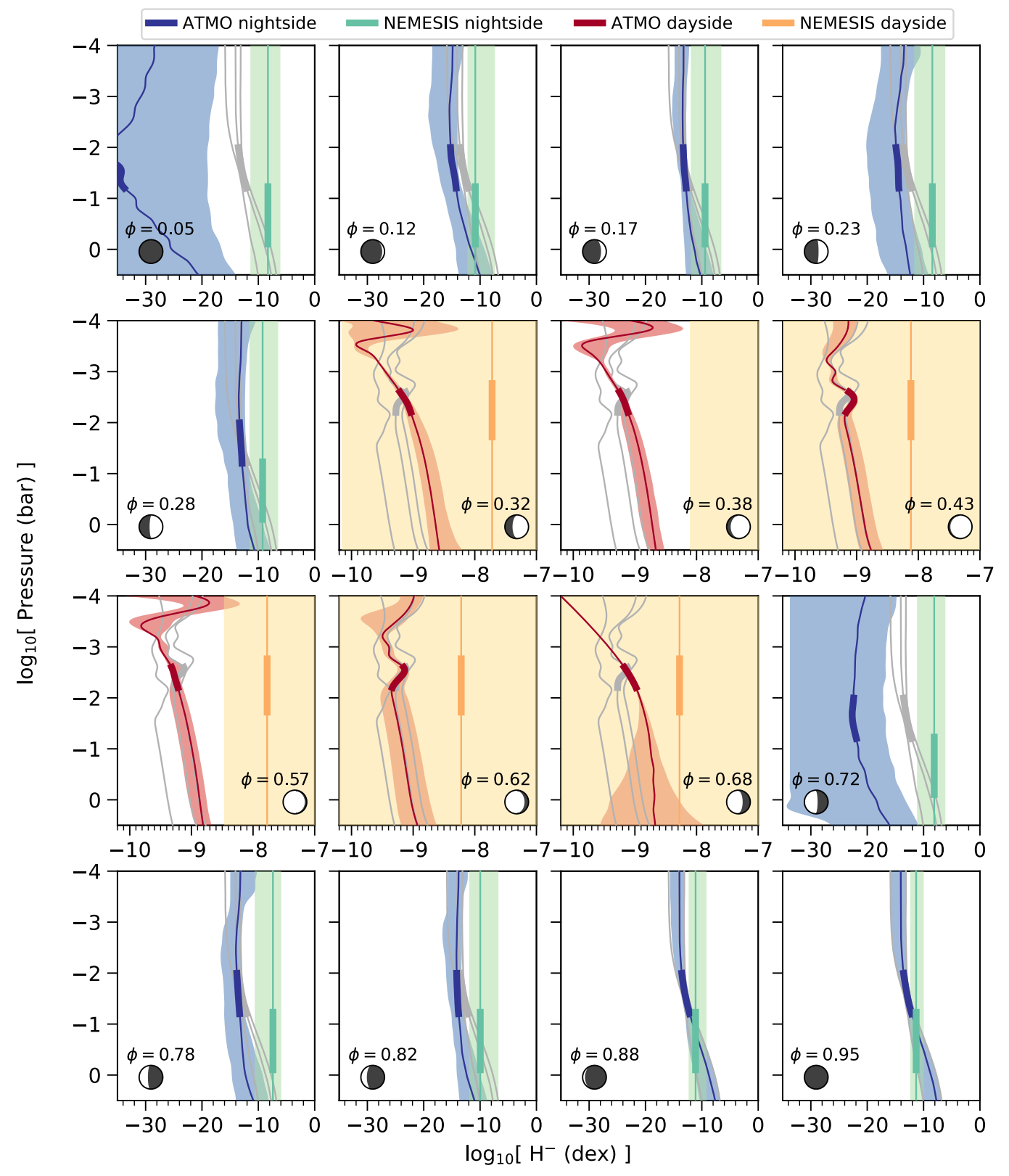

Extended Data Fig. 7. Retrieved pressure-dependent $\mathbf{H}^{-}$abundances. Same format as Extended Data Figs 5 and 6, but showing posterior distributions for the retrieved $\mathrm{H}^{-}$abundances. 


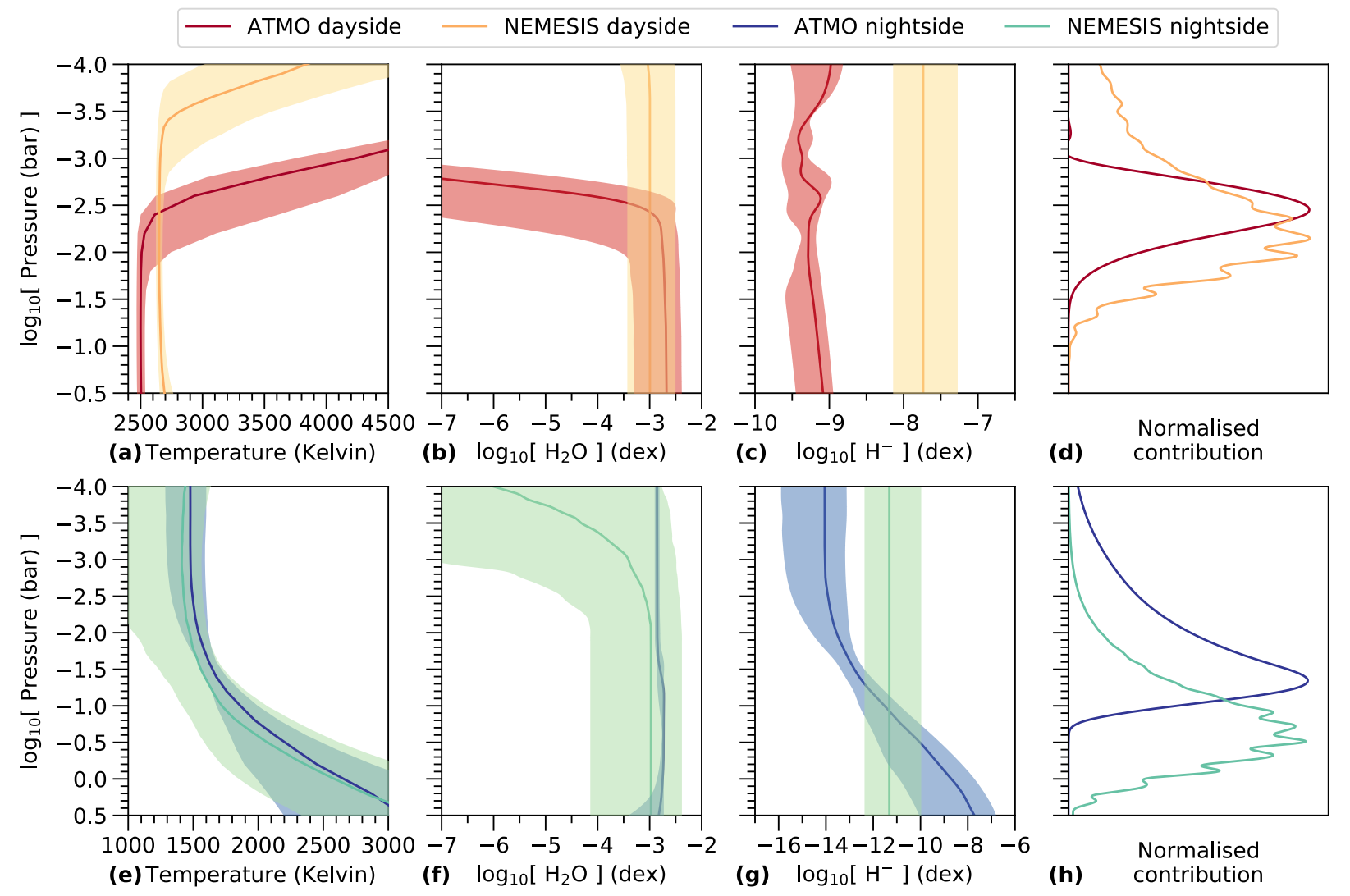

Extended Data Fig. 8. ATMO and NEMESIS “2x PT" retrievals for the fiducial dayside and nightside spectra.

Top panel shows (from left to right) the retrieved PT profiles, $\mathrm{H}_{2} \mathrm{O}$ abundances, $\mathrm{H}^{-}$abundances, and normalised contribution functions for the fiducial dayside spectrum. Bottom panel shows the same for the fiducial nightside spectrum. Same colour scheme as Extended Data Figs 5-7. 


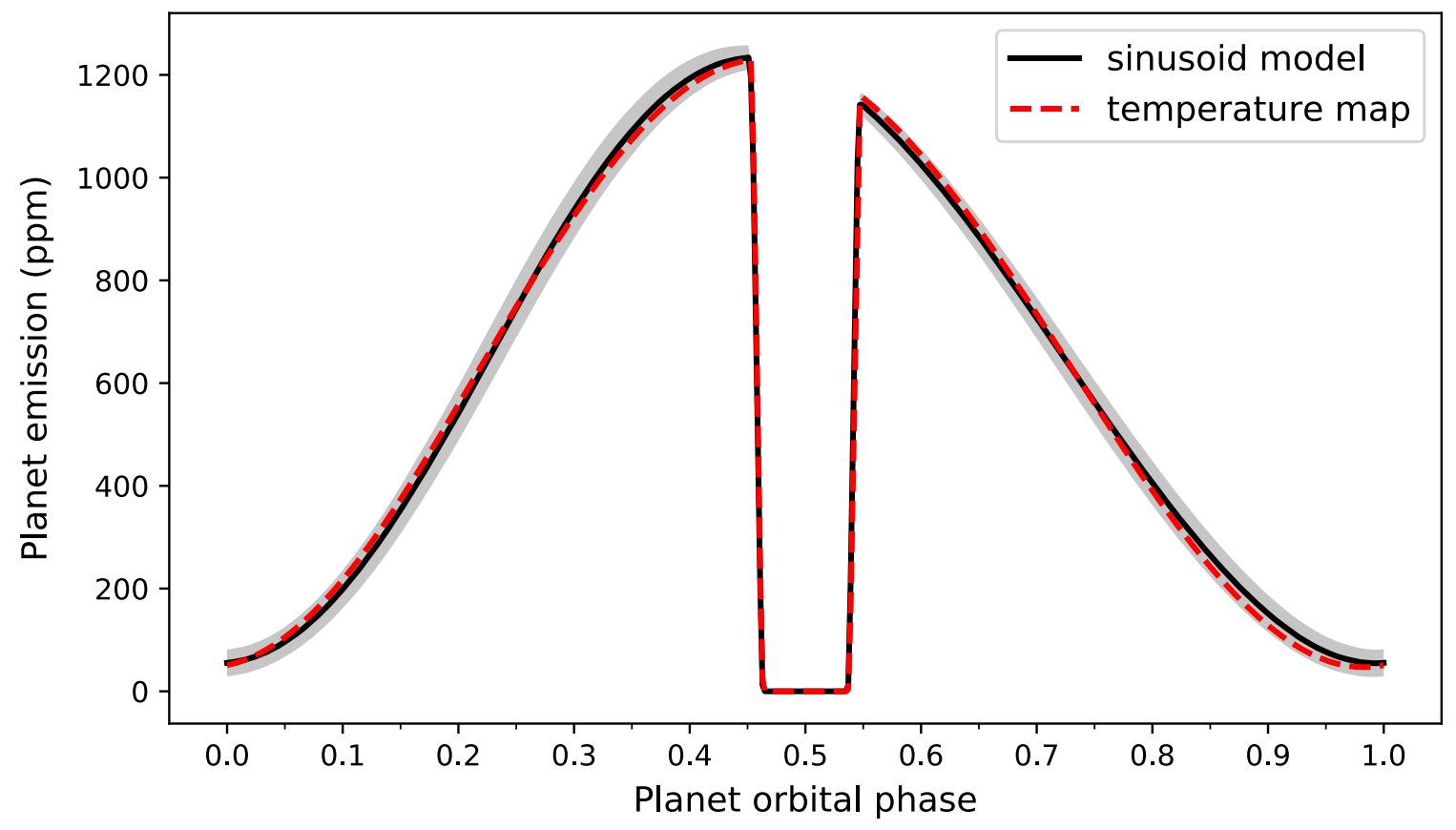

Extended Data Fig. 9. Phase curve models for the broadband planet emission of WASP-121b. Black solid line shows the median of the second-order sinusoidal models sampled by the MCMC light curve analysis and grey shading shows the corresponding standard deviation. Dashed red line shows a light curve generated by the third-order spherical harmonics temperature map described in Methods, optimised to match the second-order sinusoidal model. 


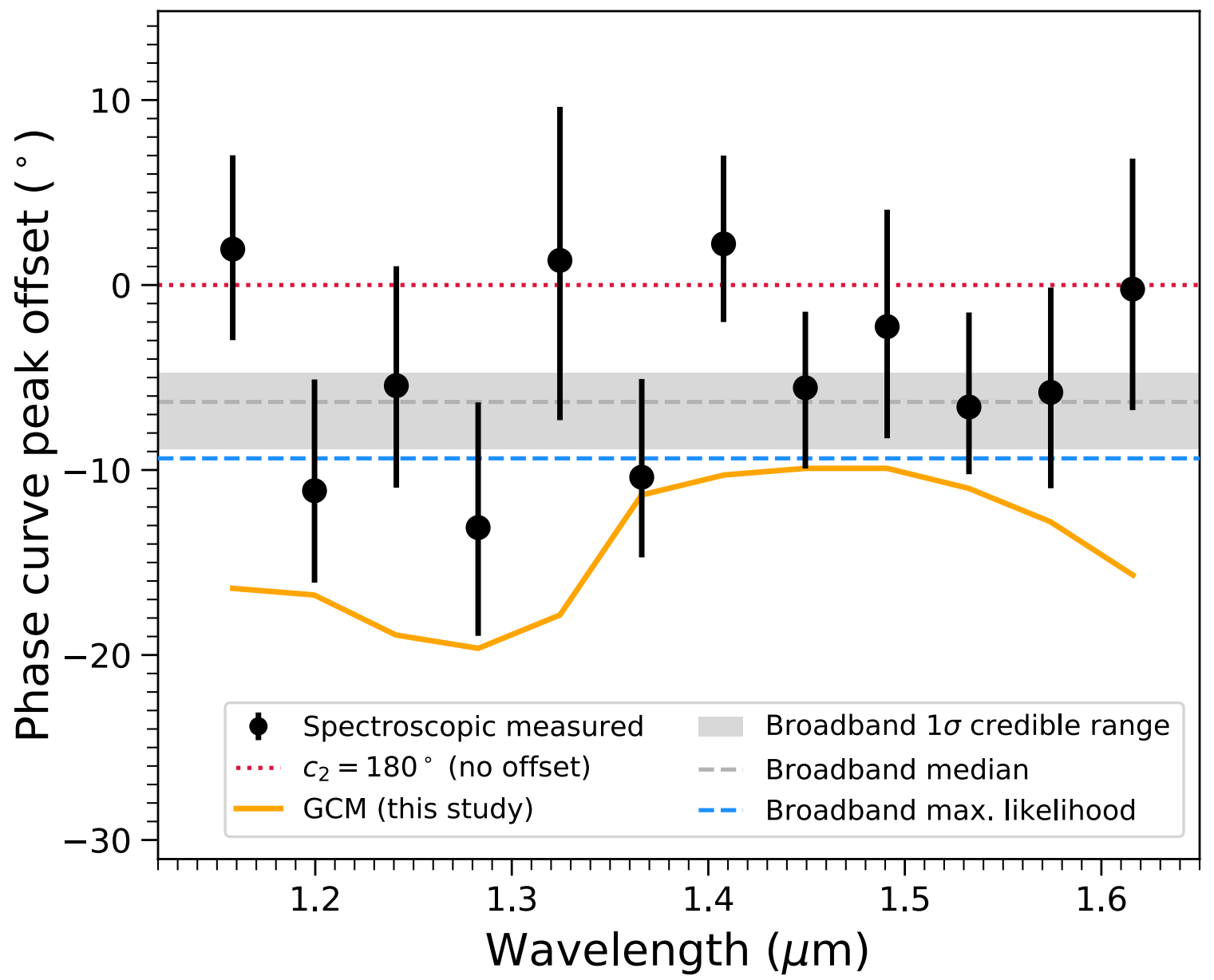

Extended Data Fig. 10. Phase curve peak offset as a function of wavelength. Black circles show posterior medians for $c_{2}-\pi$ (i.e. hot spot phase offset) obtained for each spectroscopic light curve fit, expressed in units of degrees, with error bars showing the $1 \sigma$ credible ranges. Dotted red line corresponds to the zero point of $c_{2}=\pi$ radian (i.e. $\phi=0.5$ ). Dashed grey line shows the posterior median obtained for the broadband light curve fit, with grey shading indicating the $1 \sigma$ credible range. Dashed blue line corresponds to the maximum likelihood value of $c_{2}=2.98$ radian obtained for the broadband light curve and held fixed for the final spectroscopic light curve fits. Solid orange line shows the phase curve peak offsets predicted by the GCM run for this study, assuming an atmospheric metallicity of 1x solar and no atmospheric drag. 


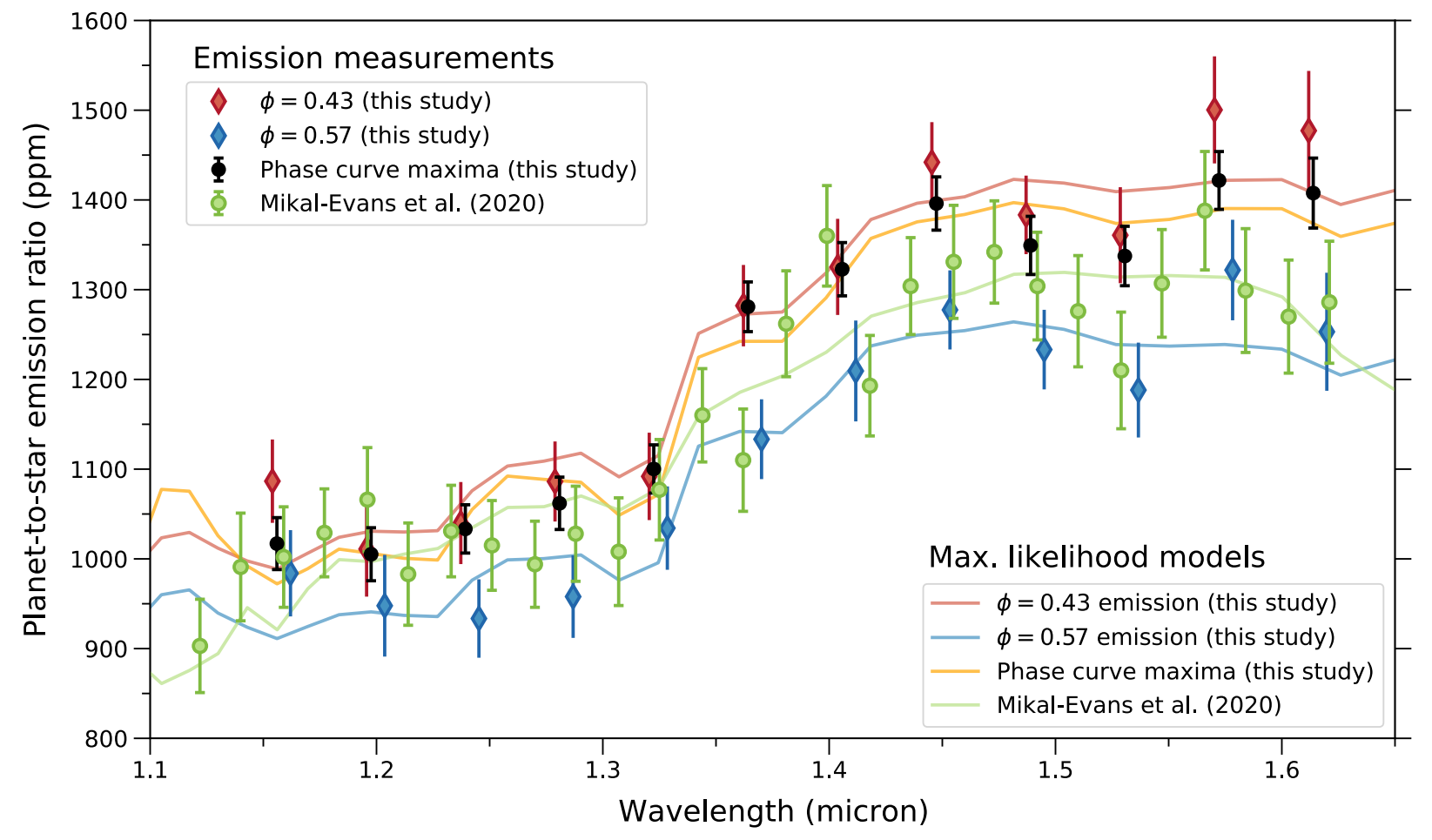

Extended Data Fig. 11. Emission spectrum of the WASP-121b dayside hemisphere. Black circles show the medians of the phase curve maxima sampled during spectroscopic light curve fitting with $1 \sigma$ error bars. Red and blue diamonds show the binned planetary flux at orbital phases $\phi=0.43$ and $\phi=0.57$, respectively, with $1 \sigma$ error bars. Green circles show the secondary eclipse spectrum published in ref. 3 with $1 \sigma$ error bars. Solid lines show the maximum likelihood models for each dataset with colour scheme described in the legend. 

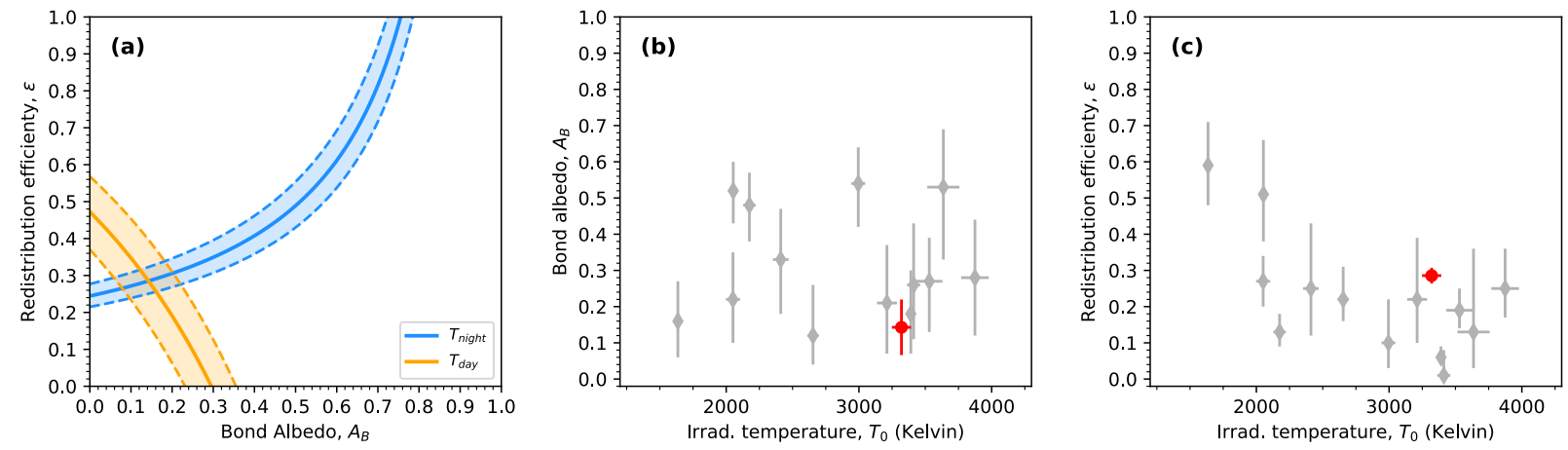

Extended Data Fig. 12. Bond albedo and heat redistribution efficiencies for WASP-121b and other irradiated substellar objects. a, Constraints obtained for the Bond albedo and heat redistribution efficiency from the dayside brightness temperature $\left(T_{\text {day }}\right)$ and nightside brightness temperature $\left(T_{\text {night }}\right)$, following the method of ref. 67. b, Grey diamonds show Bond albedos $A_{B}$ for an ensemble of irradiated substellar objects reported by ref. 68 as a function of the irradiation temperature, $T_{0}=T_{\star} \sqrt{R_{\star} / a}$. Red circle shows the result for WASP-121b obtained in the present study. Error bars give $1 \sigma$ uncertainties. $\mathbf{c}$, The same as $\mathbf{b}$ but showing the inferred heat redistribution efficiency values $\varepsilon$ as a function of irradiation temperature. 


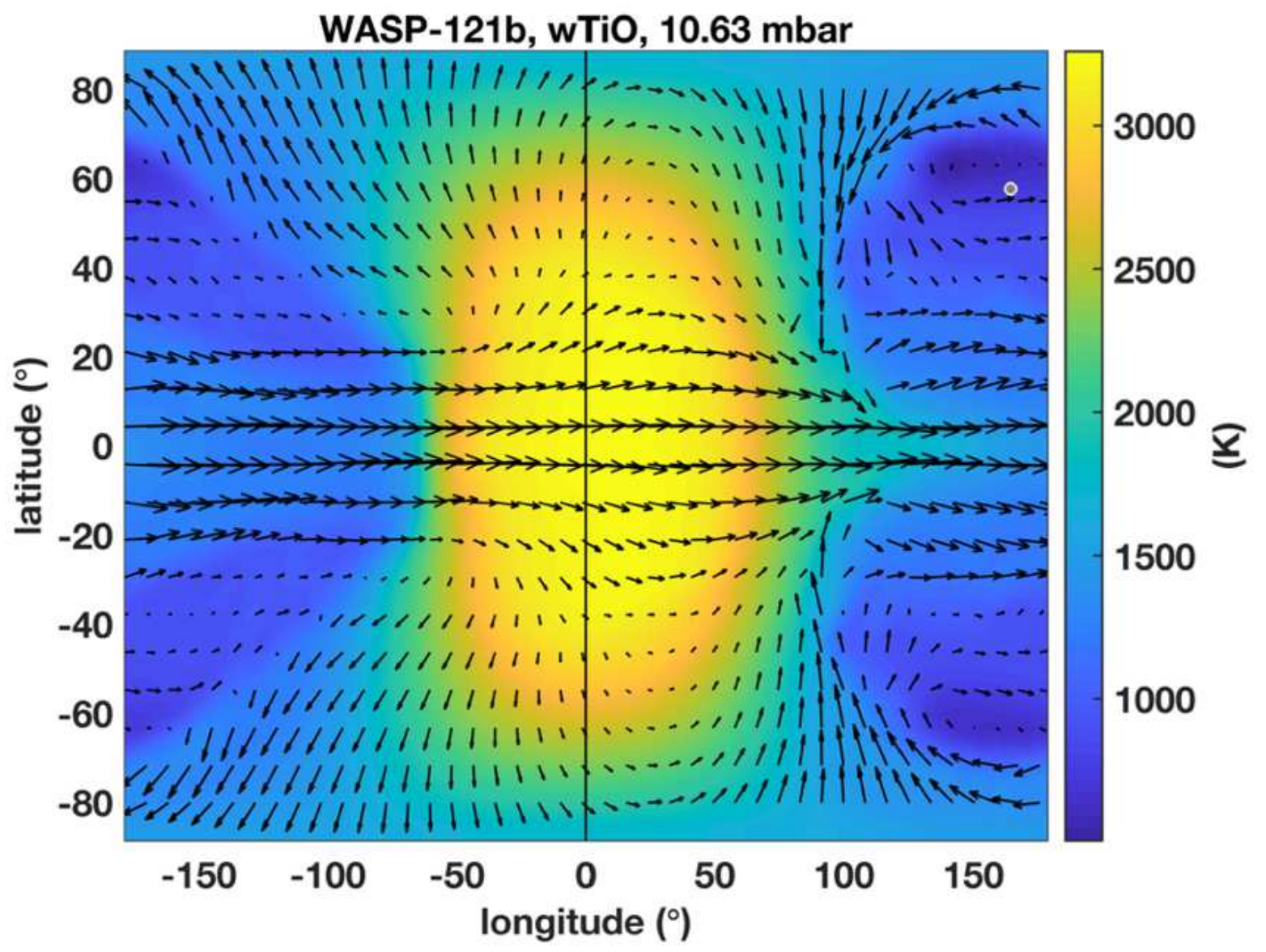

Extended Data Fig. 13. General circulation model for WASP-121b. Temperature (colour scale in Kelvin) and winds (arrows) for the SPARC GCM simulation of WASP-121b run for the present study, assuming $1 \times$ solar metallicity, chemical equilibrium, and no atmospheric drag. The vertical black line indicates the substellar longitude. 
Extended Data Table 1. Astrophysical parameters obtained from fits to the broadband light curve. Results are reported for fits adopting first-order and second-order sinusoidal models for the planetary phase variations. Values listed for free parameters are posterior medians and uncertainties give the $1 \sigma$ credible ranges. Note that inclination $i$ values have been derived from $b$ and $a / R_{\star}$. The redistribution factors $A_{F}$ have been derived from the distribution of phase curve models sampled during fitting. Although the white noise was treated as a free parameter for each dataset as described in Methods, the quoted $\chi^{2}$ and BIC values were calculated assuming photon noise to allow direct comparison of the two models.

\begin{tabular}{|c|c|c|c|}
\hline Parameter & Unit & $\begin{array}{c}\text { Sinusoid first-order } \\
\chi^{2}=1896.6 \\
\chi_{v}^{2}=2.6 \\
\operatorname{BIC}=2129.5\end{array}$ & $\begin{array}{c}\text { Sinusoid second-order } \\
\chi^{2}=1864.9 \\
\chi_{v}^{2}=2.5 \\
\mathrm{BIC}=2111.0\end{array}$ \\
\hline$R_{p} / R_{\star}$ & - & $0.12190_{-0.00008}^{+0.00009}$ & $0.12199_{-0.00009}^{+0.00009}$ \\
\hline$a / R_{\star}$ & - & $3.812_{-0.003}^{+0.004}$ & $3.785_{-0.003}^{+0.003}$ \\
\hline$b$ & - & $0.114_{-0.003}^{+0.002}$ & $0.175_{-0.002}^{+0.002}$ \\
\hline$i$ & degree & $88.29_{-0.04}^{+0.05}$ & $87.35_{-0.02}^{+0.03}$ \\
\hline$T_{p, 1}$ & JDutc & $2458191.114061_{-0.000050}^{+0.000049}$ & $2458191.114045_{-0.000048}^{+0.000054}$ \\
\hline$T_{p, 2}$ & JDuTC & $2458518.768537_{-0.000065}^{+0.000073}$ & $2458518.768609_{-0.000060}^{+0.000072}$ \\
\hline$c_{0}$ & ppm & $18_{-14}^{+28}$ & $26_{-19}^{+34}$ \\
\hline$c_{1}$ & ppm & $1195_{-29}^{+23}$ & $1158_{-40}^{+28}$ \\
\hline$c_{2}$ & radian & $3.06_{-0.05}^{+0.04}$ & $3.03_{-0.04}^{+0.03}$ \\
\hline$c_{3}$ & ppm & - & $69_{-23}^{+23}$ \\
\hline$c_{4}$ & radian & - & $4.54_{-0.02}^{+0.03}$ \\
\hline$\varepsilon_{0}$ & percent & $1.1_{-0.8}^{+1.6}$ & $1.6_{-1.2}^{+2.3}$ \\
\hline$A_{F}$ & percent & $98.6 \pm 1.9$ & $95.1 \pm 2.5$ \\
\hline$P$ & day & \multicolumn{2}{|c|}{1.2749247646 (fixed) } \\
\hline$e$ & - & \multicolumn{2}{|c|}{0 (fixed) } \\
\hline $\mathrm{u}_{1}$ & - & \multicolumn{2}{|c|}{0.072 (fixed) } \\
\hline $\mathrm{u}_{2}$ & - & \multicolumn{2}{|c|}{0.301 (fixed) } \\
\hline
\end{tabular}


Extended Data Table 2. Results of ATMO “2x PT" retrieval analyses. Values listed are the posterior medians, with uncertainties giving the $1 \sigma$ credible ranges. First row gives the results of the retrieval analysis performed for the dayside emission spectrum, with metallicity $([\mathrm{M} / \mathrm{H}])$ fitted as a free parameter. Based on the retrieved metallicity for the dayside spectrum, the metallicity was held fixed to $[\mathrm{M} / \mathrm{H}]=0.7$ (i.e. $5 \mathrm{x}$ solar) for all other orbital phases. Last row gives the results of an additional retrieval performed as a check for phase $\phi=0.95$ with $[\mathrm{M} / \mathrm{H}]$ treated as a free parameter.

\begin{tabular}{|c|c|c|c|c|}
\hline$\phi$ & $\begin{array}{c}K / R \\
\left(\mathrm{~cm}^{2} \cdot \mathrm{g}^{-1}\right)\end{array}$ & $\gamma_{1}$ & $\boldsymbol{\Psi}$ & 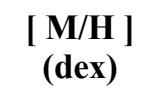 \\
\hline Dayside & $-1.78_{-0.26}^{+0.08}$ & $1.76_{-0.30}^{+0.16}$ & $1.22_{-0.01}^{+0.01}$ & $0.76_{-0.62}^{+0.30}$ \\
\hline 0.05 & $-2.99_{-0.99}^{+1.19}$ & $-0.52_{-2.16}^{+2.26}$ & $0.30_{-0.15}^{+0.18}$ & 0.7 (fixed) \\
\hline 0.12 & $-2.09_{-1.31}^{+0.91}$ & $-1.48_{-1.54}^{+2.09}$ & $0.62_{-0.14}^{+0.09}$ & 0.7 (fixed) \\
\hline 0.17 & $2.59_{-1.36}^{+1.24}$ & $-1.09_{-1.65}^{+1.45}$ & $0.73_{-0.11}^{+0.06}$ & 0.7 (fixed) \\
\hline 0.23 & $-2.33_{-1.83}^{+0.98}$ & $-0.52_{-2.03}^{+3.00}$ & $0.64_{-0.21}^{+0.11}$ & 0.7 (fixed) \\
\hline 0.28 & $-2.05_{-1.82}^{+0.91}$ & $-0.72_{-1.64}^{+3.19}$ & $0.73_{-0.21}^{+0.09}$ & 0.7 (fixed) \\
\hline 0.32 & $-2.32_{-0.45}^{+0.71}$ & $3.28_{-2.85}^{+0.43}$ & $1.31_{-0.05}^{+0.04}$ & 0.7 (fixed) \\
\hline 0.38 & $-2.17_{-0.34}^{+0.47}$ & $3.29_{-1.48}^{+0.40}$ & $1.30_{-0.05}^{+0.02}$ & 0.7 (fixed) \\
\hline 0.43 & $-1.75_{-0.14}^{+0.11}$ & $1.64_{-0.13}^{+0.09}$ & $1.25_{-0.03}^{+0.03}$ & 0.7 (fixed) \\
\hline 0.57 & $-2.04_{-0.44}^{+0.34}$ & $3.11_{-1.27}^{+0.50}$ & $1.27_{-0.04}^{+0.02}$ & 0.7 (fixed) \\
\hline 0.62 & $-1.78_{-0.16}^{+0.18}$ & $1.79_{-0.14}^{+0.12}$ & $1.22_{-0.04}^{+0.05}$ & 0.7 (fixed) \\
\hline 0.68 & $-2.77_{-1.80}^{+1.17}$ & $0.20_{-0.49}^{+0.71}$ & $1.13_{-0.14}^{+0.19}$ & 0.7 (fixed) \\
\hline 0.72 & $-2.61_{-1.32}^{+1.01}$ & $-0.99_{-1.66}^{+2.64}$ & $0.40_{-0.21}^{+0.18}$ & 0.7 (fixed) \\
\hline 0.78 & $-2.55_{-1.72}^{+1.32}$ & $-0.86_{-1.75}^{+3.51}$ & $0.70_{-0.14}^{+0.08}$ & 0.7 (fixed) \\
\hline 0.82 & $-2.67_{-1.62}^{+1.45}$ & $-1.23_{-1.65}^{+2.32}$ & $0.69_{-0.14}^{+0.07}$ & 0.7 (fixed) \\
\hline 0.88 & $-1.88_{-0.62}^{+0.53}$ & $-1.83_{-1.10}^{+1.21}$ & $0.71_{-0.08}^{+0.06}$ & 0.7 (fixed) \\
\hline 0.95 & $-1.89_{-0.56}^{+0.56}$ & $-1.90_{-1.13}^{+1.24}$ & $0.70_{-0.08}^{+0.06}$ & 0.7 (fixed) \\
\hline $\begin{array}{c}0.95 \\
(\text { free }[\mathrm{M} / \mathrm{H}])\end{array}$ & $-1.76_{-0.92}^{+0.54}$ & $-1.82_{-1.30}^{+0.98}$ & $0.70_{-0.10}^{+0.06}$ & $0.66_{-1.02}^{+0.70}$ \\
\hline
\end{tabular}


Extended Data Table 3. Astrophysical parameters obtained from the spectroscopic phase curve fits with the hot spot phase $\left(c_{2}\right)$ and higher-order terms $\left(c_{3}, c_{4}\right)$ allowed to vary as free parameters. Same format as Extended Data Tables 1 and 2.

\begin{tabular}{|c|c|c|c|c|c|c|c|c|c|c|}
\hline$\lambda(\mu \mathrm{m})$ & $u_{1}$ & $u_{2}$ & $\boldsymbol{R}_{p} / \boldsymbol{R}_{s}$ & $\begin{array}{c}c_{0} \\
(\mathbf{p p m})\end{array}$ & $\begin{array}{c}c_{1} \\
(\mathbf{p p m})\end{array}$ & $\begin{array}{c}c_{2} \\
\text { (radian) }\end{array}$ & $\begin{array}{c}c_{3} \\
(\mathbf{p p m})\end{array}$ & $\begin{array}{c}c_{4} \\
\text { (radian) }\end{array}$ & $\chi_{v}^{2}$ & BIC \\
\hline $1.137-1.179$ & 0.126 & 0.290 & $0.1209_{-0.00018}^{+0.00018}$ & $25_{-18}^{+33}$ & $980_{-48}^{+41}$ & $3.18_{-0.09}^{+0.09}$ & $72_{-36}^{+38}$ & $4.91_{-0.06}^{+0.06}$ & 1.2 & 1037.3 \\
\hline $1.179-1.220$ & 0.115 & 0.293 & $0.12119_{-0.00017}^{+0.00017}$ & $36_{-26}^{+47}$ & $972_{-52}^{+46}$ & $2.95_{-0.09}^{+0.10}$ & $51_{-30}^{+35}$ & $3.68_{-0.41}^{+0.44}$ & 1.2 & 993.0 \\
\hline $1.220-1.262$ & 0.105 & 0.298 & $0.12096_{-0.00016}^{+0.00017}$ & $31_{-23}^{+43}$ & $985_{-52}^{+45}$ & $3.05_{-0.10}^{+0.11}$ & $75_{-34}^{+32}$ & $3.93_{-0.11}^{+0.16}$ & 1.1 & 937.7 \\
\hline $1.262-1.304$ & 0.074 & 0.310 & $0.12104_{-0.00017}^{+0.00019}$ & $94_{-61}^{+73}$ & $968_{-84}^{+71}$ & $2.91_{-0.10}^{+0.12}$ & $81_{-37}^{+39}$ & $4.09_{-0.15}^{+0.17}$ & 1.3 & 1069.1 \\
\hline $1.304-1.345$ & 0.086 & 0.303 & $0.1204_{-0.00017}^{+0.00017}$ & $183_{-79}^{+72}$ & $887_{-79}^{+84}$ & $3.16_{-0.15}^{+0.14}$ & $110_{-32}^{+34}$ & $4.16_{-0.14}^{+0.15}$ & 1.2 & 1012.6 \\
\hline $1.345-1.387$ & 0.076 & 0.304 & $0.12096_{-0.00016}^{+0.00016}$ & $50_{-34}^{+56}$ & $1204_{-63}^{+50}$ & $2.96_{-0.08}^{+0.09}$ & $113_{-33}^{+34}$ & $4.05_{-0.22}^{+0.20}$ & 1.1 & 906.0 \\
\hline $1.387-1.429$ & 0.067 & 0.305 & $0.12097_{-0.00019}^{+0.00018}$ & $48_{-34}^{+54}$ & $1227_{-60}^{+47}$ & $3.18_{-0.07}^{+0.08}$ & $132_{-40}^{+37}$ & $4.21_{-0.09}^{+0.08}$ & 1.2 & 1005.2 \\
\hline $1.429-1.470$ & 0.056 & 0.306 & $0.12150_{-0.00017}^{+0.00019}$ & $33_{-25}^{+47}$ & $1336_{-54}^{+49}$ & $3.04_{-0.08}^{+0.07}$ & $94_{-36}^{+37}$ & $4.54_{-0.10}^{+0.10}$ & 1.1 & 965.4 \\
\hline $1.470-1.512$ & 0.047 & 0.306 & $0.12152_{-0.00018}^{+0.00017}$ & $129_{-74}^{+83}$ & $1207_{-87}^{+84}$ & $3.10_{-0.11}^{+0.11}$ & $79_{-34}^{+35}$ & $3.78_{-0.17}^{+0.15}$ & 1.2 & 996.7 \\
\hline $1.512-1.553$ & 0.039 & 0.302 & $0.12109_{-0.00017}^{+0.00020}$ & $39+29$ & $1303_{-57}^{+49}$ & $3.03_{-0.06}^{+0.09}$ & $43_{-27}^{+36}$ & $3.98_{-0.16}^{+0.13}$ & 1.2 & 1008.8 \\
\hline $1.553-1.595$ & 0.030 & 0.298 & $0.12165_{-0.00020}^{+0.00020}$ & $25_{-19}^{+40}$ & $1340_{-55}^{+49}$ & $3.04_{-0.09}^{+0.10}$ & $152_{-45}^{+45}$ & $5.23_{-0.08}^{+0.07}$ & 1.2 & 1019.6 \\
\hline $1.595-1.637$ & 0.024 & 0.300 & $0.12088_{-0.00022}^{+0.00021}$ & $47_{-34}^{+64}$ & $1318_{-71}^{+66}$ & $3.14_{-0.11}^{+0.12}$ & $108_{-47}^{+49}$ & $4.54_{-0.10}^{+0.12}$ & 1.4 & 1171.0 \\
\hline
\end{tabular}


Extended Data Table 4. Astrophysical parameters obtained from the spectroscopic phase curve fits with the hot spot phase $\left(c_{2}\right)$ and higher-order terms $\left(c_{3}, c_{4}\right)$ held fixed to the values determined for the broadband light curve. Same format as Extended Data Tables 1-3.

\begin{tabular}{|c|c|c|c|c|c|c|c|}
\hline$\lambda(\boldsymbol{\mu m})$ & $\boldsymbol{u}_{1}$ & $\boldsymbol{u}_{2}$ & $\boldsymbol{R}_{P} / \boldsymbol{R}_{\boldsymbol{s}}$ & $\boldsymbol{c}_{\mathbf{0}}(\mathbf{p p m})$ & $\boldsymbol{c}_{\mathbf{1}}(\mathbf{p p m})$ & $\chi_{\boldsymbol{v}}^{2}$ & BIC \\
\hline $1.137-1.179$ & 0.126 & 0.290 & $0.12108_{-0.00015}^{+0.00016}$ & $24_{-17}^{+31}$ & $990_{-41}^{+36}$ & 1.2 & 1020.6 \\
\hline $1.179-1.220$ & 0.115 & 0.293 & $0.12132_{-0.00016}^{+0.0015}$ & $29_{-21}^{+41}$ & $971_{-45}^{+36}$ & 1.2 & 977.1 \\
\hline $1.220-1.262$ & 0.105 & 0.298 & $0.12111_{-0.00014}^{+0.00015}$ & $24_{-17}^{+38}$ & $1004_{-42}^{+35}$ & 1.1 & 921.5 \\
\hline $1.262-1.304$ & 0.074 & 0.310 & $0.12115_{-0.00017}^{+0.00017}$ & $82_{-52}^{+66}$ & $979_{-67}^{+57}$ & 1.3 & 1055.2 \\
\hline $1.304-1.345$ & 0.086 & 0.303 & $0.12115_{-0.00016}^{+0.00016}$ & $154_{-71}^{+72}$ & $947_{-76}^{+75}$ & 1.2 & 1001.7 \\
\hline $1.345-1.387$ & 0.076 & 0.304 & $0.12112_{-0.00015}^{+0.00015}$ & $49_{-34}^{+55}$ & $1229_{-58}^{+44}$ & 1.1 & 897.0 \\
\hline $1.387-1.429$ & 0.067 & 0.305 & $0.12113_{-0.00016}^{+0.00016}$ & $38_{-27}^{+45}$ & $1281_{-51}^{+41}$ & 1.2 & 999.9 \\
\hline $1.429-1.470$ & 0.056 & 0.306 & $0.12154_{-0.00016}^{+0.0016}$ & $28_{-20}^{+39}$ & $1363_{-46}^{+38}$ & 1.1 & 949.3 \\
\hline $1.470-1.512$ & 0.047 & 0.306 & $0.12165_{-0.00017}^{+0.00018}$ & $95_{-59}^{+67}$ & $1255_{-75}^{+64}$ & 1.2 & 985.7 \\
\hline $1.512-1.553$ & 0.039 & 0.302 & $0.12121_{-0.00018}^{+0.00016}$ & $28_{-21}^{+44}$ & $1302_{-47}^{+43}$ & 1.2 & 992.0 \\
\hline $1.553-1.595$ & 0.030 & 0.298 & $0.12148_{-0.00018}^{+0.00018}$ & $36_{-26}^{+48}$ & $1382_{-59}^{+44}$ & 1.2 & 1004.8 \\
\hline $1.595-1.637$ & 0.024 & 0.300 & $0.12095_{-0.00023}^{+0.00020}$ & $48_{-34}^{+56}$ & $1354_{-67}^{+56}$ & 1.4 & 1155.9 \\
\hline
\end{tabular}


Extended Data Table 5. Heat redistribution factors and fit metrics for the two suites of spectroscopic light curve fits. Heat redistribution factors $A_{F}$ were derived in the same manner as described for Extended Data Table 1. Fit metrics have been reproduced here from Extended Data Tables 3 and 4 to ease comparison between the two suites of phase curve fits.

\begin{tabular}{|c|c|c|c|c|c|c|}
\hline & \multicolumn{3}{|c|}{$c_{2}, c_{3}, c_{4}$ allowed to vary } & \multicolumn{3}{|c|}{$c_{2}, c_{3}, c_{4}$ held fixed } \\
\hline$\lambda(\mu \mathrm{m})$ & $A_{F}(\%)$ & $\chi_{v}^{2}$ & BIC & $A_{F}(\%)$ & $\chi_{v}^{2}$ & BIC \\
\hline 1.137-1.179 & $94.1 \pm 3.1$ & 1.2 & 1037.3 & $93.5 \pm 2.6$ & 1.2 & 1020.6 \\
\hline $1.179-1.220$ & $95.2 \pm 3.8$ & 1.2 & 993.0 & $92.9 \pm 2.9$ & 1.2 & 977.1 \\
\hline $1.220-1.262$ & $95.4 \pm 3.4$ & 1.1 & 937.7 & $93.5 \pm 3.0$ & 1.1 & 921.5 \\
\hline 1.262-1.304 & $88.9 \pm 5.7$ & 1.3 & 1069.14 & $88.7 \pm 5.0$ & 1.3 & 1055.2 \\
\hline 1.304-1.345 & $81.8 \pm 6.6$ & 1.2 & 1012.6 & $82.8 \pm 6.0$ & 1.2 & 1001.7 \\
\hline $1.345-1.387$ & $93.7 \pm 3.4$ & 1.1 & 906.0 & $92.9 \pm 3.4$ & 1.1 & 897.0 \\
\hline $1.387-1.429$ & $94.7 \pm 3.3$ & 1.2 & 1005.2 & $93.9 \pm 2.8$ & 1.2 & 999.9 \\
\hline $1.429-1.470$ & $94.6 \pm 2.6$ & 1.1 & 965.4 & $94.9 \pm 2.1$ & 1.2 & 949.3 \\
\hline $1.470-1.512$ & $90.0 \pm 5.5$ & 1.2 & 996.7 & $89.9 \pm 4.3$ & 1.2 & 985.7 \\
\hline $1.512-1.553$ & $96.4 \pm 2.9$ & 1.2 & 1008.8 & $94.6 \pm 2.5$ & 1.4 & 992.0 \\
\hline $1.553-1.595$ & $90.6 \pm 2.8$ & 1.2 & 1019.6 & $94.4 \pm 2.7$ & 1.2 & 1004.8 \\
\hline $1.595-1.637$ & $94.0 \pm 3.6$ & 1.4 & 1171.0 & $93.5 \pm 3.2$ & 1.2 & 1155.9 \\
\hline
\end{tabular}


Extended Data Table 6. Measured planetary emission spectrum at each orbital phase. Values are in units of ppm and quoted uncertainties give the $1 \sigma$ credible ranges. Continued on next page.

\begin{tabular}{|c|c|c|c|c|c|c|}
\hline & \multicolumn{6}{|c|}{ Spectroscopic wavelength channel (micron) } \\
\hline$\phi$ & 1.137-1.179 & $1.179-1.220$ & $1.220-1.262$ & $1.262-1.304$ & 1.304-1.345 & 1.345-1.387 \\
\hline 0.05 & $28 \pm 52$ & $68 \pm 60$ & $30 \pm 47$ & $71 \pm 48$ & $201 \pm 54$ & $70 \pm 48$ \\
\hline 0.12 & $182 \pm 51$ & $228 \pm 63$ & $234 \pm 50$ & $387 \pm 57$ & $313 \pm 56$ & $310 \pm 56$ \\
\hline 0.17 & $425 \pm 53$ & $420 \pm 58$ & $369 \pm 50$ & $437 \pm 54$ & $475 \pm 56$ & $472 \pm 59$ \\
\hline 0.23 & $605 \pm 59$ & $539 \pm 64$ & $526 \pm 56$ & $594 \pm 51$ & $738 \pm 54$ & $659 \pm 48$ \\
\hline 0.28 & $705 \pm 65$ & $665 \pm 55$ & $758 \pm 53$ & $762 \pm 48$ & $804 \pm 50$ & $891 \pm 48$ \\
\hline 0.32 & $861 \pm 49$ & $900 \pm 61$ & $851 \pm 48$ & $892 \pm 51$ & $1004 \pm 48$ & $1053 \pm 48$ \\
\hline 0.38 & $909 \pm 48$ & $975 \pm 52$ & $1005 \pm 45$ & $1066 \pm 46$ & $1088 \pm 49$ & $1266 \pm 45$ \\
\hline 0.43 & $1087 \pm 46$ & $1011 \pm 53$ & $1040 \pm 46$ & $1086 \pm 45$ & $1092 \pm 49$ & $1282 \pm 45$ \\
\hline 0.57 & $984 \pm 48$ & $948 \pm 57$ & $934 \pm 44$ & $958 \pm 46$ & $1034 \pm 46$ & $1133 \pm 44$ \\
\hline 0.62 & $813 \pm 54$ & $815 \pm 54$ & $829 \pm 45$ & $843 \pm 45$ & $895 \pm 48$ & $1018 \pm 48$ \\
\hline 0.68 & $594 \pm 57$ & $588 \pm 53$ & $636 \pm 50$ & $781 \pm 52$ & $731 \pm 58$ & $833 \pm 59$ \\
\hline 0.72 & $508 \pm 57$ & $525 \pm 56$ & $537 \pm 54$ & $577 \pm 51$ & $679 \pm 51$ & $688 \pm 53$ \\
\hline 0.78 & $360 \pm 53$ & $382 \pm 67$ & $401 \pm 53$ & $479 \pm 60$ & $495 \pm 53$ & $493 \pm 45$ \\
\hline 0.82 & $233 \pm 55$ & $297 \pm 65$ & $268 \pm 50$ & $292 \pm 48$ & $388 \pm 61$ & $325 \pm 51$ \\
\hline 0.88 & $226 \pm 61$ & $115 \pm 57$ & $161 \pm 46$ & $243 \pm 59$ & $335 \pm 55$ & $136 \pm 54$ \\
\hline 0.95 & $82 \pm 49$ & $48 \pm 54$ & $93 \pm 45$ & $162 \pm 50$ & $219 \pm 47$ & $86 \pm 47$ \\
\hline
\end{tabular}


Extended Data Table 6 (continued from previous page).

\begin{tabular}{|c|c|c|c|c|c|c|}
\hline & \multicolumn{6}{|c|}{ Spectroscopic wavelength channel (micron) } \\
\hline$\phi$ & 1.387-1.429 & 1.429-1.470 & $1.470-1.512$ & $1.512-1.553$ & 1.553-1.595 & 1.595-1.637 \\
\hline 0.05 & $45 \pm 60$ & $56 \pm 52$ & $169 \pm 54$ & $50 \pm 57$ & $78 \pm 62$ & $19 \pm 71$ \\
\hline 0.12 & $253 \pm 60$ & $321 \pm 50$ & $327 \pm 52$ & $304 \pm 58$ & $309 \pm 60$ & $257 \pm 87$ \\
\hline 0.17 & $495 \pm 58$ & $514 \pm 41$ & $524 \pm 51$ & $542 \pm 64$ & $523 \pm 70$ & $568 \pm 73$ \\
\hline 0.23 & $772 \pm 61$ & $752 \pm 50$ & $815 \pm 52$ & $704 \pm 55$ & $746 \pm 67$ & $715 \pm 69$ \\
\hline 0.28 & $894 \pm 57$ & $971 \pm 47$ & $968 \pm 58$ & $936 \pm 65$ & $913 \pm 68$ & $1078 \pm 66$ \\
\hline 0.32 & $1169 \pm 68$ & $1133 \pm 55$ & $1194 \pm 57$ & $1113 \pm 64$ & $1173 \pm 63$ & $1160 \pm 71$ \\
\hline 0.38 & $1234 \pm 58$ & $1336 \pm 45$ & $1243 \pm 47$ & $1241 \pm 56$ & $1351 \pm 56$ & $1253 \pm 69$ \\
\hline 0.43 & $1325 \pm 54$ & $1442 \pm 45$ & $1383 \pm 44$ & $1361 \pm 53$ & $1500 \pm 60$ & $1477 \pm 67$ \\
\hline 0.57 & $1209 \pm 56$ & $1277 \pm 44$ & $1233 \pm 44$ & $1188 \pm 53$ & $1322 \pm 56$ & $1253 \pm 66$ \\
\hline 0.62 & $980 \pm 57$ & $1114 \pm 45$ & $1099 \pm 46$ & $1106 \pm 55$ & $1094 \pm 54$ & $1136 \pm 65$ \\
\hline 0.68 & $935 \pm 59$ & $880 \pm 48$ & $935 \pm 55$ & $878 \pm 58$ & $962 \pm 55$ & $893 \pm 74$ \\
\hline 0.72 & $681 \pm 59$ & $723 \pm 51$ & $708 \pm 54$ & $611 \pm 59$ & $670 \pm 70$ & $708 \pm 61$ \\
\hline 0.78 & $479 \pm 59$ & $528 \pm 51$ & $530 \pm 55$ & $482 \pm 60$ & $475 \pm 61$ & $461 \pm 78$ \\
\hline 0.82 & $272 \pm 64$ & $327 \pm 57$ & $42 \pm 56$ & $403 \pm 55$ & $301 \pm 61$ & $394 \pm 76$ \\
\hline 0.88 & $254 \pm 61$ & $190 \pm 51$ & $226 \pm 56$ & $124 \pm 57$ & $239 \pm 65$ & $219 \pm 74$ \\
\hline 0.95 & $89 \pm 56$ & $86 \pm 46$ & $116 \pm 50$ & $74 \pm 55$ & $121 \pm 59$ & $125 \pm 69$ \\
\hline
\end{tabular}


Extended Data Table 7. Dayside emission spectrum derived from the distribution of phase curve maxima. Quoted values were derived from the distribution of phase curve maxima sampled during spectroscopic phase curve fitting with quoted uncertainties corresponding to the $1 \sigma$ credible ranges.

\begin{tabular}{|c|c|}
\hline$\lambda(\mu \mathrm{m})$ & Emission (ppm) \\
\hline $1.137-1.179$ & $1017 \pm 29$ \\
\hline $1.179-1.220$ & $1005 \pm 30$ \\
\hline $1.220-1.262$ & $1033 \pm 27$ \\
\hline $1.262-1.304$ & $1062 \pm 29$ \\
\hline $1.304-1.345$ & $1100 \pm 27$ \\
\hline $1.345-1.387$ & $1281 \pm 28$ \\
\hline $1.387-1.429$ & $1323 \pm 30$ \\
\hline $1.429-1.470$ & $1396 \pm 30$ \\
\hline $1.470-1.512$ & $1349 \pm 32$ \\
\hline $1.512-1.553$ & $1338 \pm 33$ \\
\hline $1.553-1.595$ & $1422 \pm 32$ \\
\hline $1.595-1.637$ & $1408 \pm 39$ \\
\hline
\end{tabular}


Extended Data Table 8. Emission spectrum $\chi^{2}$ fit statistics at each orbital phase for the ATMO " $2 \mathrm{x}$ PT" retrievals, NEMESIS "2x PT" retrievals, and blackbody fits. As described in Methods, the ATMO retrievals assumed chemical equilibrium, the NEMESIS retrievals assumed no constraints for the atmospheric chemistry, and the blackbody fits assumed the planet radiates isothermally. Inferred brightness temperatures with $1 \sigma$ uncertainties are also reported for the blackbody fits in the last column.

\begin{tabular}{|c|c|c|c|c|c|c|c|}
\hline \multirow[b]{2}{*}{$\phi$} & \multicolumn{2}{|c|}{$\begin{array}{l}\text { ATMO 2x PT }(\boldsymbol{v}=\mathbf{9}) \\
\text { Equilibrium chemistry } 5 \mathrm{x} \text { solar }\end{array}$} & \multicolumn{2}{|c|}{$\begin{array}{l}\text { NEMESIS 2x PT }(v=6) \\
\text { Unconstrained chemistry }\end{array}$} & \multicolumn{3}{|c|}{$\begin{array}{l}\text { Blackbody }(v=11) \\
\text { Isothermal radiation }\end{array}$} \\
\hline & $\chi^{2}$ & $\chi_{v}^{2}$ & $\chi^{2}$ & $\chi_{v}^{2}$ & $\chi^{2}$ & $\chi_{v}^{2}$ & $T_{b}$ (Kelvin) \\
\hline 0.05 & 11.4 & 1.3 & 11.5 & 2.3 & 13.8 & 1.3 & $1555 \pm 52$ \\
\hline 0.12 & 7.3 & 0.8 & 13.3 & 2.7 & 19.7 & 1.8 & $1972 \pm 22$ \\
\hline 0.17 & 2.8 & 0.3 & 5.3 & 1.1 & 16.9 & 1.5 & $2190 \pm 16$ \\
\hline 0.23 & 9.2 & 1.0 & 13.2 & 2.6 & 30.8 & 2.8 & $2366 \pm 13$ \\
\hline 0.28 & 4.5 & 0.5 & 14.0 & 2.8 & 15.5 & 1.4 & $2505 \pm 11$ \\
\hline 0.32 & 4.3 & 0.5 & 12.2 & 2.4 & 23.1 & 2.1 & $2622 \pm 10$ \\
\hline 0.38 & 9.5 & 1.1 & 12.1 & 2.4 & 35.1 & 3.2 & $2699 \pm 9$ \\
\hline 0.43 & 11.7 & 1.3 & 19.8 & 4.0 & 25.6 & 2.3 & $2749 \pm 8$ \\
\hline 0.57 & 8.1 & 0.9 & 19.2 & 3.8 & 31.4 & 2.9 & $2670 \pm 9$ \\
\hline 0.62 & 3.7 & 0.4 & 12.6 & 2.5 & 20.5 & 1.9 & $2580 \pm 9$ \\
\hline 0.68 & 7.0 & 0.8 & 10.5 & 2.1 & 16.1 & 1.5 & $2461 \pm 12$ \\
\hline 0.72 & 8.1 & 0.9 & 9.6 & 1.9 & 32.1 & 2.9 & $2331 \pm 13$ \\
\hline 0.78 & 4.0 & 0.4 & 3.6 & 0.7 & 25.6 & 2.3 & $2178 \pm 16$ \\
\hline 0.82 & 7.8 & 0.9 & 9.1 & 1.8 & 16.0 & 1.5 & $2022 \pm 20$ \\
\hline 0.88 & 15.1 & 1.7 & 14.2 & 2.8 & 26.5 & 2.4 & $1838 \pm 28$ \\
\hline 0.95 & 7.5 & 0.8 & 7.6 & 1.5 & 16.0 & 1.5 & $1651 \pm 38$ \\
\hline Mean & 7.6 & 0.8 & 11.7 & 2.3 & 22.8 & 2.1 & \\
\hline Median & 7.6 & 0.8 & 12.1 & 2.4 & 21.8 & 2.0 & \\
\hline
\end{tabular}


Extended Data Table 9. Fit metrics demonstrating nightside emission detection. Results are reported for the fiducial ATMO "2x PT" retrievals assuming chemical equilibrium with $5 \mathrm{x}$ solar metallicity at the ten phases for which retrievals were performed for the nightside emission while holding the dayside contribution fixed. Also reported are fit statistics obtained in a similar manner except that the nightside emission was fixed to zero (i.e. $\Phi_{n}=0$ ). The Bayes factors reported in the final column are computed as $\exp (-\Delta \mathrm{BIC} / 2)$. The final row gives values for the ensemble of phase-resolved emission spectra listed in this table.

\begin{tabular}{|c|c|c|c|c|c|c|}
\hline & $\begin{array}{c}\text { ATMO 2x PT (v=9) } \\
\text { Fiducial model } \\
\text { i.e. nonzero nightside } \\
\text { emission allowed }\end{array}$ & $\begin{array}{c}\text { ATMO 2x PT (v= 12) } \\
\text { Fiducial model for dayside } \\
\text { contribution but nightside } \\
\text { emission fixed to zero }\end{array}$ & \multicolumn{2}{|c|}{$\begin{array}{c}\text { Model comparison } \\
\text { Favouring nightside emission }\end{array}$} \\
\hline $\boldsymbol{\phi}$ & $\chi^{2}$ & BIC & $\chi^{2}$ & BIC & $\Delta$ BIC & Bayes Factor \\
\hline 0.05 & 11.4 & 18.8 & 11.6 & 11.6 & 7.2 & 0.03 \\
\hline 0.12 & 7.3 & 14.8 & 17.3 & 17.3 & -2.5 & 3.5 \\
\hline 0.17 & 2.8 & 10.3 & 20.8 & 20.8 & -10.5 & 193.6 \\
\hline 0.23 & 9.2 & 16.7 & 17.9 & 17.9 & -1.3 & 1.9 \\
\hline 0.28 & 4.5 & 12.0 & 11.5 & 11.5 & 0.5 & 0.8 \\
\hline 0.72 & 8.1 & 15.6 & 8.5 & 8.5 & 7.1 & 0.03 \\
\hline 0.78 & 4.0 & 11.4 & 20.6 & 20.6 & -9.1 & 96.9 \\
\hline 0.82 & 7.8 & 15.2 & 20.7 & 20.7 & -5.5 & 15.7 \\
\hline 0.88 & 15.1 & 22.5 & 50.1 & 50.1 & -27.6 & $9.9 \times 10^{5}$ \\
\hline 0.95 & 7.5 & 15.0 & 57.5 & 57.5 & -42.5 & $1.7 \times 10^{9}$ \\
\hline All $\phi$ & $\mathbf{7 7 . 7}$ & $\mathbf{2 2 1 . 3}$ & $\mathbf{2 3 6 . 5}$ & $\mathbf{3 8 0 . 1}$ & $-\mathbf{1 5 8 . 8}$ & $\mathbf{3 . 0} \times \mathbf{1 0} \mathbf{1 0}^{\mathbf{3 4}}$ \\
\hline
\end{tabular}

